Homology, Homotopy and Applications, vol.4(2), 2002, pp.83-116

\title{
AN ANALOGUE OF HOLONOMIC D-MODULES ON SMOOTH VARIETIES IN POSITIVE CHARACTERISTICS
}

\author{
RIKARD BÖGVAD \\ (communicated by Larry Lambe)
}

Abstract

In this paper a definition of a category of modules over the ring of differential operators on a smooth variety of finite type in positive characteristics is given. It has some of the good properties of holonomic D-modules in zero characteristic. We prove that it is a Serre category and that it is closed under the usual D-module functors, as defined by Haastert. The relation to the similar concept of F-finite modules, introduced by Lyubeznik, is elucidated, and several examples, such as etale algebras, are given.

\section{To Jan-Erik Roos on his sixty-fifth birthday}

\section{Introduction}

The theory of $\mathcal{D}$-modules in positive characteristics has recently received attention, in several contexts. There is for example, in commutative algebra, the result by Hunecke-Sharp [8], which says that local cohomology modules, $H_{I}(R)$, where $I \subset R$ is an ideal in a regular ring over a field of characteristic $\mathrm{p}$, have finite Bass numbers. The proof makes essential, though implicit, use of the fact that these modules are $\mathcal{D}$ modules. This result was later generalized by Lyubeznik $[\mathbf{9}, \mathbf{1 0}]$, to on the one hand, a proof of the finiteness of Bass numbers for local cohomology modules in characteristic zero, by ordinary characteristic zero $\mathcal{D}$-module theory, and on the other hand to the nice concept of F-finite modules, of which more below. Other examples are the applications to the theory of tight closure due to K.Smith [12], and the proof by K.Smith and van der Berg $[\mathbf{1 3}]$ of the fact that the ring of differential operators of an invariant ring $S(V)^{G}$ is a simple ring, in positive characteristics(where $G$ is a linearly reductive group, with a finite dimensional representation $V$, and $S(V)$ the symmetric algebra on $V$ ). The corresponding result is still unknown in characteristic zero. So $\mathcal{D}$-modules are very useful objects, even in positive characteristics.

Let $X$ be a smooth variety over a field $k$ of positive characteristic p. and $\mathcal{D}_{X}$ the ring of (Grothendieck) differential operators on $X$. In this paper we will define and study a certain nice category of $\mathcal{D}_{X}$-modules, called filtration holonomic modules.

Received March 7, 2001, revised January 11, 2002; published on July 12, 2002.

2000 Mathematics Subject Classification: 32C38, 14F10, 16S32

Key words and phrases: ring of differential operators, positive characteristics, holonomic, F-finite module.

(c) 2002, Rikard Bögvad. Permission to copy for private use granted. 
These were introduced (in a stronger version) in [3]. There they were used to prove that local cohomology modules $H_{Z}^{i}\left(\mathcal{O}_{X}\right), Z$ a subvariety of $X$, have finite decomposition series as $\mathcal{D}_{X}$-modules, and to study the socle of these modules, inspired by corresponding results in characteristic zero. By the adjective nice, is meant in particular that they form a Serre subcategory of the category of all $\mathcal{D}_{X}$-modules. In addition all modules in it have finite decomposition series, and it includes several classes of important modules, like the local cohomology modules of the structure sheaf.

To motivate the concept, consider what happens for an affine smooth variety $X=\operatorname{spec} R$ over a field $k$ of characteristic zero. The ring of differential operators on $X$ may be filtered by the degree of a differential operator, and the associated graded ring is a finitely generated commutative ring. Using the Hilbert polynomial, this makes it possible and easy to develop a theory of "growth" of $\mathcal{D}$-modules. The modules with minimal growth are called holonomic modules. The growth of them turns out to be precisely equal to the growth of the $\mathcal{D}$-module $R$. These modules play an important part in $\mathcal{D}$-module theory $([\mathbf{2}, \mathbf{4}])$. In positive characteristic $\mathrm{p}$ the ring of differential operators of a smooth variety is however non-Noetherian, and hence the tools of Noetherian commutative rings are unavailable here. However it is possible to use the idea of growth in a different way. We will sketch the idea. Suppose for simplicity that $R=k\left[x_{1}, \ldots, x_{n}\right]$, where $k$ is a field of characteristic $p$. The idea is then to use the well known Morita type characterization of a module $M$ over the ring of differential operators $\mathcal{D}_{R}$. It says that such modules are precisely those modules for which there is a series of $k\left[x^{p^{r}}\right]$-modules $M^{(r)}$ and (compatible) isomorphisms

$$
\theta_{r}: F^{* r} M^{(r)}:=k[x] \otimes_{k\left[x^{p^{r}}\right]} M^{(r)} \cong M, r \geqslant 0,
$$

where $F$ is the Frobenius map. Let $V_{r}$ be the vectorspace of monomials of degree strictly less than $p^{r}$ in each variable. Then $k[x] \cong V_{r} \otimes_{k} k\left[x^{p^{r}}\right]$, and $F^{* r} M^{(r)}=$ $V_{r} \otimes_{k} M^{(r)}$. Then the archetype of a filtration holonomic module is a module that may be generated by a sequence of finite dimensional subspaces $A_{r} \subset M^{(r)}$, i. e. such that $\left.M=\cup_{\geqslant 0} \theta_{r}\left(V_{r} \otimes A_{r}\right)\right)$, where the dimensions of $A_{r}$ has a common upper bound. For example, if $M=k[x]$, then $\theta_{r}: F^{* r} M=M$ is just the ordinary canonical isomorphism $k[x] \otimes_{k\left[x^{p^{r}}\right]} k\left[x^{p^{r}}\right] \cong k[x]$, and letting the 1-dimensional vectorspaces $A_{r}$ be defined as $A_{r}=k \subset k\left[x^{p^{r}}\right]=M^{(r)}$ we have $\theta_{r}\left(V_{r} \otimes k\right)=V_{r} \subset k[x]$, and $M=\cup r_{\geqslant 0} V_{r}$. So $k[x]$ is (unsurprisingly) a filtration holonomic module.

However the definition above, given in [3], should be modified. This is because it seems impossible to so prove that extensions of filtration holonomic modules in this sense also are filtration holonomic. Instead of demanding that the dimensions of the vector spaces $A_{i}$ in the definition above should have a common bound, the modification consists of the weaker condition that a certain weighted dimension $t\left(A_{i}\right)$ associated to each vector subspace $A_{i}$ of a $\mathcal{D}_{X}$-module should be finite (Definition 3.2). We redo and develope several results from [3] , using this more general concept. (A motivating example for the modified definition is given at the end of section 3.1.)

Our main result is the following, whose first part is immediate from the local version Theorem 4.2, and whose proof is contained in sections 2-4. 
Theorem 1.1. If $X$ is a smooth variety of finite type over a field $k$ of characterisic $p$, then the category of filtration holonomic modules is closed under $\mathcal{D}_{X}$-module extensions, submodules and quotient modules. Every filtration holonomic module has a finite decomposition series.

We also prove that the property is preserved under the usual functors:

Theorem 1.2. If $X$ is a smooth variety of finite type over a field $k$ of characterisic $p$, then the category of filtration holonomic modules preserved by direct and inverse images, and local cohomology.

A different finiteness condition for $\mathcal{D}_{X}$-modules $-F$-finite modules -has recently been introduced by Lyubeznik [10], building on the work by Huneke and Sharp [8]. It has similar properties: e.g. $F$-finite modules also have finite decomposition series, as $\mathcal{D}_{X}$-modules, and local cohomology modules of the structure sheaf are $F$-finite. We analyze to some extent in this paper the relation between these two concepts and show in particular that $F$-finite modules are filtration holonomic (for a smooth variety of finite type over a perfect field), but that the converse does not hold, in general. This difference is mainly due to the fact that built into the concept of a $F$ finite module $M$, is that the module is a so-called $F$-module, i.e. that as $R$-modules $F^{*} M \cong M$. As was described above this is equivalent (by iteration and Moritaequivalence) to the fact that $M$ is a $\mathcal{D}_{X}$-module, with the extra condition that $M \cong$ $M^{(r)}, r=1,2, \ldots$ This is rather restrictive, and has for example the consequence that a $\mathcal{D}_{X}$-module extension of $F$-finite modules is not necessarily $F$-finite. It should however be noted that $F$-finite modules may be used in a more general situation, e.g. complete regular rings, while the concept of filtration holonomic modules is bound to the condition that the variety is of finite type.

Finally we give several examples of filtration holonomic modules. First, each étale algebra $E$ over $R=k\left[x_{1}, \ldots, x_{n}\right]$, considered as a $\mathcal{D}_{R}$-module has this property. The proof is rather involved, but constructive, meaning that it is possible to get bounds on the length of a finite decomposition series of $R$. This example was already described in $[\mathbf{3}]$, but the proof given there was deficient(as kindly pointed out by M.Kaneda). Other examples are in the class of $\mathcal{O}_{X}$-coherent $\mathcal{D}_{X}$-modules. It is trivial to prove that modules in this class have a finite decomposition series but unlike the situation in characteristic zero, complicated to prove that they are filtration holonomic, and in fact we only succeed for modules which correspond to étale sheaves, though we conjecture it to be true in general. An example is given which supports this conjecture.

I would like to thank Torsten Ekedahl and Gennady Lyubeznik for several discussions on these topics; Masaharu Kaneda for carefully puncturing several attempts at proofs, in particular the proof of the filtration holonomicity of the étale extensions. Thanks are also due to the referee for very useful comments. 


\section{Filtrations on vector subspaces of $\mathcal{D}$-modules}

\subsection{The idea}

Assume that $X=\mathbf{A}^{n}=\operatorname{spec} k\left[x_{1}, \ldots, x_{n}\right]$. Since $X$ is affine there is no need to work with sheaves. Let, as in $2.4, V_{i}$ be the $k$-vector subspace of $k[x]$ generated by all monomials $x_{1}^{i_{1}} \ldots x_{n}^{i_{n}}$, where $i_{j}<p^{i}$, for all $j=1, \ldots, n$.

To start the analysis of the growth of a $\mathcal{D}_{X}$-module, note that Cartier's lemma, in the form of Proposition 2.4 gives the following way of characterizing a submodule of a $\mathcal{D}_{X}$-module.

Proposition 2.1. Suppose that $\mathcal{M}$ is a $\mathcal{D}_{X}$-module and that the $k[x]$-module $\mathcal{N} \subset$ $\mathcal{M}$ is a union

$$
\mathcal{N}=\bigcup_{j \geqslant 0} V_{j} A_{j}
$$

where $A_{j} \subset \mathcal{N}^{(j)}$, and that furthermore an arbitrary element in $\mathcal{N}$ is contained in all except a finite number of the vector spaces $V_{j} A_{j}$. Then $\mathcal{N}$ is a $\mathcal{D}_{X}$-submodule.

Conversely every $\mathcal{D}_{X}$-module with a countable number of generators, e. g. a submodule of a finitely generated $\mathcal{D}_{X}$-module $\mathcal{M}$, may be described as a union (1), for some sequence of finite-dimensional vectorspaces $A_{j}, j \geqslant 0$.

Proof. Recall that $(2.4) \mathcal{D}_{X}^{(j)}=\mathcal{O}_{X} \Delta_{\mathbf{A}^{n}}^{(j)}$. Since $\mathcal{N}$ is an $\mathcal{O}_{X^{-}}$-module, note that it suffices to show that $\mathcal{N}$ is a $\Delta_{\mathbf{A}^{n}}^{(j)}$-module, for all $j>0$. Assume that $\delta \in \Delta_{\mathbf{A}^{n}}^{(j)}$. Clearly (see 2.4), $V_{j_{0}} A_{j_{0}}$ is a $\Delta_{\mathbf{A}^{n}}^{\left(j_{0}\right)}$-submodule of $\mathcal{N}$ and, by hypothesis, there is to each $f \in \mathcal{N}$ a $j_{0} \geqslant j$ such that $f \in V_{j_{0}} A_{j_{0}}$. Hence $\delta(f) \in V_{j_{0}} A_{j_{0}}$.

Conversely, every $\mathcal{D}_{X}$-module $\mathcal{M}$ with a countable number of generators, has countable dimension as a vector space over $k$, and so contains finite-dimensional vectorspaces $B_{j}, j>0$ such that $B_{j} \subset B_{j+1}$ and $\cup_{j>0} B_{j}=\mathcal{M}$. Then, by Proposition 2.4, there are finite-dimensional vectorspaces $A_{j} \subset \mathcal{M}^{(j)}$ such that $B_{j} \subset V_{j} A_{j}$. Since any element in $\mathcal{M}$ is contained in almost all $B_{j}$, this is also true of almost all $V_{j} A_{j}$.

It should be emphasized that $\mathcal{M}^{(j)}$ is always thought of as a submodule of the module $\mathcal{M}$, and that the inclusion is not $\mathcal{O}_{X}$-linear but really an inclusion $\mathcal{M}^{(j)} \subset$ $F_{*} \mathcal{M}$.

The idea of the finiteness condition introduced in [3], described in the introduction, and there called filtration holonomic modules, is then that minimal "growth" of a module is obtained when the vector space dimension of the sequence $A_{i}$, for a filtration of type (1) for the module, is bounded. This definition will be modified below, so as to make it easier to handle, but unfortunately necessitating more technical details.

We will give an example to motivate the increase in technical difficulty. Recall the description of the idea behind filtration holonomic modules, given in the intro-

duction. The problem with this definition arises when one tries to prove that there is some finiteness condition on an extension of two filtration holonomic module. We will describe a $\mathcal{D}_{k[x]}$-module $\mathcal{M}$ which is a $\mathcal{D}_{k[x]}$-module extension of $k[x]$ with itself. 
Let $\mathcal{M}$ be a free $k[x]$-module on 2 generators $s_{1}, s_{2}$, and similarily let $\mathcal{M}^{(i)}, i \geqslant 0$ be a free $k\left[x^{\left(p^{i}\right)}\right]$-module on 2 generators $s_{1}^{(i)}, s_{2}^{(i)}$. Define

$$
\Theta_{i}: \mathcal{M}^{(i+1)} \rightarrow \mathcal{M}^{(i)}
$$

by $\Theta_{i}\left(s_{1}^{(i+1)}\right)=s_{1}^{(i)}$ and $\Theta_{i}\left(s_{2}^{(i+1)}\right)=s_{2}^{(i)}+g_{i} s_{1}^{(i)}$ where $g_{i} \in k\left[x^{p^{i}}\right]$ is arbitrary. This is an inclusion, and we may think of all $\mathcal{M}^{(i)}$ as contained in $\mathcal{M}$. In particular $s_{1}^{(i)}=s_{1}^{(0)}$ and

$$
s_{2}^{(i)}=s_{2}^{(0)}+\left(\sum_{m=0}^{i} g_{m}\right) s_{1}^{(0)} .
$$

This inverse system gives a $\mathcal{D}_{k[x]}$-module structure on $\mathcal{M}$. It contains $k[x] s_{1}^{(0)}$ which is isomorphic to $k[x]$, and it projects to $k[x] s_{2}^{(0)}$, also isomorphic to $k[x]$. If all $g_{i}=0$ then $\mathcal{M}$ is the direct sum of these two $\mathcal{D}_{k[x]}$-modules. To estimate the growth it suffices to note that, in this case, the union of all $V_{i}\left(k s_{1}^{(i)}+k s_{2}^{(i)}\right)=V_{i}\left(k s_{1}^{(0)}+k s_{2}^{(0)}\right)$ equals $\mathcal{M}$. But suppose now that the degree of $G_{i}:=\sum_{m=0}^{i} g_{m}$ grows very quickly; for example that $\operatorname{deg} G_{i} / p^{i}$ goes to infinity. Then the union of $V_{i}\left(k s_{1}^{(i)}+k s_{2}^{(i)}\right)$ will not equal $\mathcal{M}$, and it is not difficult to prove that there are no sequence of finitedimensional vector spaces $A_{i} \subset \mathcal{M}^{(i)}$ such that the union of all $V_{i} A_{i}$ is $\mathcal{M}$. This means that $\mathcal{M}$ is not filtration holonomic according to the naive definition in the introduction and [3]. However clearly

$$
V_{i}\left(k s_{1}^{(0)}+k s_{2}^{(0)}\right) \subset B_{i}:=V_{i+d(i)}\left(k s_{1}^{(0)}\right)+V_{i}\left(k s_{2}^{(0)}\right),
$$

where $d(i)$ is chosen so that $G_{i} \in V_{i+d(i)}$. In some sense then, the extension $M$ still has a "growth" as a $\mathcal{D}_{k[x]}$-module that is the same as $k[x]$, and is with respect to this "generated" by a sequence of two-dimensional vector spaces. This intuition will be worked out in the rest of the section.

\subsection{Vector subspaces of the type $V_{i} A$}

The following lemma on how to handle elementary vector space operations of vector subspaces of the type $V_{i} A$ where $A \subset \mathcal{M}^{(i)}$, is an immediate consequence of the flatness of the Frobenius, see Proposition 2.4.

Lemma 2.2.1. Suppose that $\mathcal{M}$ is a $\mathcal{D}_{X}$-module and that the vector spaces $A$ and $B$ are contained in $\mathcal{M}^{(i)}$. Then the canonical map

$$
V_{i} \otimes_{k} A \rightarrow V_{i} A
$$

is an isomorphism. Also

$$
V_{i} A \cap V_{i} B=V_{i}(A \cap B)
$$

and

$$
V_{i} A+V_{i} B=V_{i}(A+B) .
$$

Furthermore if

$$
V_{i} A \subset V_{i} B,
$$


then

$$
A \subset B .
$$

It follows also, in particular, from (2) and (3), that $V_{i} A=V_{i} B$ implies that $A=B$.

The following obvious lemma is also included here for handy reference. If the vector subspace $A \subset k[x]$, denote by $A^{\left[p^{r}\right]}$ the image of $k \otimes_{k} A \rightarrow k \otimes_{k} k[x] \rightarrow k[x]$, where the second map is $r$ th iterate of the relative Frobenius $F_{x / \text { speck }}$. For example, $V_{1}^{\left[p^{r}\right]}$ is the vector subspace of $k[x]$ generated by all monomials $x^{i}$, where $i_{j}=p^{r} k_{j}$, and $0 \leqslant k_{j}<p$, for $j=1, \ldots, n$. A calculation of degrees then gives

Lemma 2.2.2. $V_{k+1}=V_{k}\left(V_{1}\right)^{\left[p^{k}\right]}$, if $k \geqslant 0$, or more generally

$$
V_{j}=V_{i}\left(V_{j-i}\right)^{\left[p^{i}\right]},
$$

if $j \geqslant i$.

\subsection{A canonical filtration}

In this subsection we will study certain filtrations which are defined for arbitrary vector subspaces of $\mathcal{D}$-modules, and which will be used to express the finiteness condition for D-modules given below, in definition 4.0.1.

Suppose that $A$ is an arbitrary vector sub space of $\mathcal{M}$. An immediate consequence of (4) of Lemma 3.2.1 is that there is, for each i, an unique maximal vector subspace $\tau^{i}(A) \subset A$, containing all vector spaces of the form $V_{i} B$, where $B \subset \mathcal{M}^{(i)}$. Furthermore, by the same lemma, $\tau^{i}(A)=V_{i} \Phi^{i}(A)$, where $\Phi^{i}(A) \subset \mathcal{M}^{(i)}$ is uniquely determined. By Lemma 3.2.2, $\tau^{i+1}(A)=V_{i+1} \Phi^{i+1}(A)=V_{i} V_{1}^{\left[p^{i}\right]} \Phi^{i+1}(A) \subset \tau^{i}(A)$, since $V_{1}^{\left[p^{i}\right]} \Phi^{i+1}(A) \subset \mathcal{M}^{(i)}$. Hence there is a canonical filtration

$$
A=\tau^{0}(A) \supset \tau^{1}(A) \supset \ldots,
$$

which is finite if $A$ is finite dimensional.

We now want to introduce a measure $t(A)$ of how complicated this filtration is. The desired result is given in Definition 3.2 below. Note that $A \supset \tau^{1}(A)=$ $V_{1} \Phi^{1}(A)$, and that, as above, $\tau^{i}(A)=V_{i} \Phi^{i}(A) \supset \tau^{i+1}(A)=V_{i+1} \Phi^{i+1}(A)=$ $V_{i}\left(V_{1}\right)^{\left[p^{i}\right]} \Phi^{i+1}(A)$, by lemma 3.2.2. Hence by lemma 3.2.1, $\Phi^{i}(A) \supset\left(V_{1}\right)^{\left[p^{i}\right]} \Phi^{i+1}(A)$. Suppose now that $\tau^{k+1}(A)=0$ and define then

$$
\begin{aligned}
t(A): & =\left|A / \tau^{1}(A)\right|+\left|\Phi^{1}(A) /\left(V_{1}\right)^{[p]} \Phi^{2}(A)\right|+\ldots \\
& +\left|\Phi^{k-1}(A) /\left(V_{1}\right)^{\left[p^{k-1}\right]} \Phi^{k}(A)\right|+\left|\Phi^{k}(A)\right|,
\end{aligned}
$$

where $|A|$ denotes the dimension of the vector space $A$. Since $\left|V_{i}\right|=p^{i n}$, we have

$$
\left|\tau^{i}(A)\right|=\left|V_{i} \Phi^{i}(A)\right|=p^{i n}\left|\Phi^{i}(A)\right|,
$$

and hence

$$
\left|\Phi^{i}(A)\right|=p^{-i n}\left|\tau^{i}(A)\right|, \text { and }\left|\left(V_{1}\right)^{\left[p^{i}\right]} \Phi^{i+1}(A)\right|=p^{-i n}\left|\tau^{i+1}(A)\right|
$$

so that 


$$
\left|\Phi^{i}(A) /\left(V_{1}\right)^{\left[p^{i}\right]} \Phi^{i+1}(A)\right|=p^{-i n}\left(\left|\tau^{i}(A)\right|-\left|\tau^{i+1}(A)\right|\right)=p^{-i n}\left|\tau^{i}(A) / \tau^{i+1}(A)\right| .
$$

Thus $t(A)$ may also be described as

$$
\begin{aligned}
t(A) & =\sum_{i \geqslant 0} p^{-i n}\left|\tau^{i}(A) / \tau^{i+1}(A)\right|= \\
& =|A|-\sum_{i>0} p^{(i-1) n}\left(1-p^{-n}\right)\left|\tau^{i}(A)\right|,
\end{aligned}
$$

since $\tau^{k+1}(A)=0$.

This type of measure actually makes some sense for any filtration $F$. of $A$, which is contained in the $\tau$-filtration.

Definition and Lemma 2.2. Let $A=F^{0} \supset F^{1} \supset \ldots \supset F^{k+1}=0$ be a filtration of the finite-dimensional vector space $A$, such that $F^{i} \subset \tau^{i}(A)$. Define

$$
t_{F}(A):=\sum_{i \geqslant 0} p^{-n i}\left|F^{i} / F^{i+1}\right| .
$$

Then also

$$
t_{F}(A)=\left|F^{0}\right|-\sum_{i \geqslant 1} p^{-n(i-1)}\left(1-p^{-n}\right)\left|F^{i}\right| .
$$

If $F \cdot \subset G$, are two such filtrations, then $t_{F}(A) \geqslant t_{G}(A)$, with equality if and only if the two filtrations coincide. Furthermore, if $F$. has the property that $F^{i}=V_{i} \Phi^{i}(F)$, for some vectorspace $\Phi^{i}(F) \in M^{(i)}$, then

$$
t_{F}(A)=\left|A / F^{1}(A)\right|+\left|\Phi^{(1)} /\left(V_{1}\right)^{p} \Phi^{(2)}\right|+\ldots+\left|\Phi^{(k-1)} /\left(V_{1}\right)^{p^{k-1}} \Phi^{(k)}\right|+\left|\Phi^{(k)}\right| .
$$

Proof. The equality of the three expressions for $t_{F}(A)$ is clear by the argument preceding the lemma, while the inequality is immediate from the alternate expression (7) of $t_{F}(A)$, noting that $\left|F_{0}\right|=\left|G_{0}\right|=|A|$ and $\left|F_{i}\right| \leqslant\left|G_{i}\right|$, since $F_{i} \subset G_{i}$ by assumption.

Hence $t(A)$ may also be characterized as the minimal value of $t_{F}(A)$, for all filtrations $F \cdot \subset \tau^{\cdot}(A)$.

$\mathcal{M}^{(i)}$ is a $\mathcal{D}_{X^{\left(p^{i}\right)}}$-module where $X^{\left(p^{i}\right)}=\operatorname{spec} k\left[x^{p^{i}}\right]$. Since $k\left[x^{p^{i}}\right] \cong k[x]$, we may do the preceding for $A \subset \mathcal{M}^{(i)}$, and obtain a canonical filtration etc, denoted by $\tau_{i}^{j}(A)=\left(V_{j}\right)^{\left[p^{i}\right]} \Phi_{i}^{j}(A)$, where $\Phi_{i}^{j}(A) \subset \mathcal{M}^{(i+j)}$, and corresponding to this a measure $t_{i}(A)$. Note then the following property of $t(A)$, which follows from Lemma 3.2.2.

Lemma 2.3.1. If $A \subset \mathcal{M}^{(i)}$, then $V_{i} \tau_{i}^{j}(A)=\tau^{i+j}\left(V_{i} A\right)$, if $j \geqslant 0$, and $\tau^{k}\left(V_{i} A\right)=0$, if $k<i$, hence $t\left(V_{i} A\right)=t_{i}(A)$.

There is also another characterization of $t(A)$, which gives the reason why we are interested in it. It says that $t(A)$ is a measure on the minimal dimension of a vector space needed to "generate" in the special sense described in i) below. 
Proposition 2.3. For a finite-dimensional vector subspace $A$ of $\mathcal{M}$ and an integer $K$ the following statements are equivalent:

i) There are vector subspaces $B_{i} \subset \mathcal{M}^{(i)}, i \geqslant 0$ such that

$$
A=\sum_{i \geqslant 0} V_{i} B_{i} \text { and } \sum_{i \geqslant 0}\left|B_{i}\right| \leqslant K
$$

ii) $t(A) \leqslant K$.

Proof. Assume condition (i), and define a filtration

$$
F^{i}:=\Sigma_{j \geqslant i} V_{j} B_{j}, i \geqslant 0 .
$$

Then clearly

$$
F^{i}=V_{i}\left(\Sigma_{j \geqslant i}\left(V_{j-i}\right)^{\left[p^{j}\right]} B_{j}\right) \subset \tau^{i}(A),
$$

and so by the preceding lemma $t_{F}(A) \geqslant t(A)$. But, letting $\Phi^{i}(F):=\Sigma_{j \geqslant i}\left(V_{j-i}\right)^{\left[p^{j}\right]} B_{j}$ so that $F^{i}=V_{i} \Phi^{i}(F)$, we have

$$
\begin{gathered}
F^{i} / F^{i+1}=\left(V_{i} \Phi^{i}(F)\right) /\left(V_{i}\left(V_{1}\right)^{\left[p^{i}\right]} \Phi^{i+1}(F)\right) \cong V_{i} \otimes\left(\sum_{j \geqslant i} V_{j-i}^{\left[p^{i}\right]} B_{j} / \sum_{j \geqslant i+1} V_{j-i}^{\left[p^{i}\right]} B_{j}\right) \\
\cong V_{i} \otimes\left(B_{i} /\left(B_{i} \cap\left(\sum_{j \geqslant i+1} V_{j-i}^{\left[p^{i}\right]} B_{j}\right)\right)\right)
\end{gathered}
$$

and hence $\left|\Phi^{i}(F) /\left(V_{1}\right)^{\left[p^{i}\right]} \Phi^{i+1}(F)\right| \leqslant\left|B_{i}\right|$, and so $t(A) \leqslant t_{F}(A) \leqslant \Sigma_{i}\left|B_{i}\right| \leqslant K$.

Conversely, assume that $\tau^{k+1}(A)=0$ and choose by descending recursion, for each $i$ such that $0 \leqslant i \leqslant k$, starting with $B_{k}:=\Phi^{k}(A)$, a vector subspace $B_{i}$ of $\Phi^{i}(A)$ which is mapped isomorphically by the quotient map onto $\Phi^{i}(A) /\left(V_{1}\right)^{\left[p^{i}\right]} \Phi^{i+1}(A)$, Then, by induction on the length $k$ of the filtration, $A=\sum_{i \geqslant 0} V_{i} B_{i}$ and by definition $K \geqslant t(A)=\sum_{i \geqslant 0}\left|B_{i}\right|$.

\subsection{The behaviour of the canonical filtration with respect to submod- ules and quotient modules}

The measure $t(A)$ defined above does not behave well with respect to vector subspaces. For example $t\left(V_{1}\right)=1$, but $t(B)=|B|$ for any proper vector subspace $B \subset V_{1}$. However, the situation is better when intersecting with a $\mathcal{D}_{X}$-submodule.

Proposition 2.4. Suppose that $A \subset \mathcal{M}$ is a finite-dimensional vector subspace of the $\mathcal{D}_{X}$-module $\mathcal{M}$, and that $\mathcal{N} \subset \mathcal{M}$ is a $\mathcal{D}_{X}$-submodule. Then the filtration $\tau$ of the preceding section satisfies

$$
\mathcal{N} \cap \tau^{i}(A)=\tau_{\mathcal{N}}^{i}(A \cap \mathcal{N}),
$$

and

$$
t_{\mathcal{N}}(A \cap \mathcal{N}) \leqslant t(A)
$$

(By $\tau_{\mathcal{N}}$ is meant the canonical filtration with respect to vector subspaces of $\mathcal{N}$.) Equality holds in the last inequality if and only if $A \cap \mathcal{N}=A$. 
Proof. Since the subspace $\tau_{\mathcal{N}}^{i}(A \cap \mathcal{N})=V_{i} \Phi_{\mathcal{N}}^{i}(A \cap \mathcal{N})$, where $\Phi_{\mathcal{N}}^{i}(A \cap \mathcal{N}) \subset \mathcal{N}^{(i)} \subset$ $\mathcal{M}^{(i)}$, it is by definition contained in $\tau^{i}(A)$. To prove the opposite inclusion, note that $\mathcal{N}=V_{i} \mathcal{N}^{(i)}$ and hence by Lemma 3.2.1.,

$$
\mathcal{N} \cap \tau^{i}(A)=V_{i} \mathcal{N}^{(i)} \cap V_{i} \Phi^{i}(A)=V_{i}\left(\mathcal{N}^{(i)} \cap \Phi^{i}(A)\right) \subset \tau_{\mathcal{N}}^{i}(A \cap \mathcal{N}) .
$$

This proves the first part of the lemma. (Note that it follows from the proof that $\left.\Phi_{\mathcal{N}}^{i}(A \cap \mathcal{N})=\mathcal{N} \cap \Phi^{i}(A)=\mathcal{N}^{(i)} \cap \Phi^{i}(A).\right)$

Denote the graded module associated to the $\tau$-filtration by $g r_{\tau}$. Then the preceding result implies that $g r_{\tau}(A \cap N) \subset g r_{\tau}(A)$, and hence

$$
p^{-n i} \mid\left(\tau_{\mathcal{N}}^{i}(A \cap N) / \tau_{\mathcal{N}}^{i+1}(A \cap N)\left|\leqslant p^{-n i}\right| \tau^{i}(A) / \tau^{i+1}(A) \mid,\right.
$$

and then summing over $i \geqslant 0$ gives that $t(A \cap \mathcal{N}) \leqslant t(A)$. Equality clearly implies that $g r_{\tau}(A \cap N)=g r_{\tau}(A)$ and this, by a general result on graded modules associated to finite filtrations, implies that $A \cap \mathcal{N}=A$.

Quotient modules are slightly worse.

Proposition 2.5. Suppose that $A \subset \mathcal{M}$ is a finite-dimensional vector subspace of the $\mathcal{D}_{X}$-module $\mathcal{M}$, and that $\mathcal{N} \subset \mathcal{M}$ is a $\mathcal{D}_{X}$-submodule. Then the filtration $\tau$ above satisfies

$$
\tau^{i}(A)+\mathcal{N} \subset \tau_{\mathcal{M} / \mathcal{N}}^{i}(A+\mathcal{N})
$$

and

$$
t_{\mathcal{M} / \mathcal{N}}(A+\mathcal{N}) \leqslant t(A)
$$

with equality implying (but not being implied by) $A \cap \mathcal{N}=0$.

There is not equality in (8) in general. An example: $k$ has characteristic $2, A=$ $k e \oplus k(x e+f)$ is a vector subspace of the module $\mathcal{M}:=k[x] e \oplus k[x] f$, which is generated by the two horizontal sections $e, f$, and $\mathcal{N}:=k[x] f$. Then $V_{1}=k \oplus k x$ and $\tau^{1}(A)=0$, so that $\tau^{1}(A)+\mathcal{N}=\mathcal{N}$ but $\tau_{\mathcal{M} / \mathcal{N}}^{1}(A+\mathcal{N})=A+\mathcal{N}$.

Proof. The inclusion (1) is clear, since

$$
\tau^{i}(A)+\mathcal{N}=V_{i} \Phi^{i}(A)+\mathcal{N}=V_{i}\left(\Phi^{i}(A)+\mathcal{N}\right),
$$

and

$$
\Phi^{i}(A)+\mathcal{N} \subset(\mathcal{M} / \mathcal{N})^{(i)} .
$$

(By the Morita-eqiuvalence $(\mathcal{M} / \mathcal{N})^{(i)}=\mathcal{M}^{(i)} / \mathcal{N}^{(i)}$ Then using Lemma 3.3 .1 on the filtration $F^{i}:=\tau^{i}(A)+\mathcal{N}$ of $\mathcal{M} / \mathcal{N}$ gives that $t_{F}(A+\mathcal{N}) \geqslant t_{\mathcal{M} / \mathcal{N}}(A+\mathcal{N})$. But the obvious map $\theta: g r_{\tau}(A) \rightarrow g r_{F}(A+\mathcal{N})$ is surjective and hence

$$
t(A)=\sum_{i \geqslant 0} p^{-n i}\left|g r_{\tau}^{i}(A)\right| \geqslant \sum_{i \geqslant 0} p^{-n i}\left|g r_{F}^{i}(A+\mathcal{N})\right|=t_{F}(A+\mathcal{N}) .
$$

(By definition 3.3.1.) This gives the inequality. The argument also shows that equality holds in (9), if and only if both the condition that $\theta$ is an isomorphism and the condition that $t_{F}(A+\mathcal{N})=t_{\mathcal{M} / \mathcal{N}}(A+\mathcal{N})$ are fulfilled. However, the first of this conditions holds if and only if $A \cap \mathcal{N}=0$. 
There is an exact sequence of graded modules

$$
0 \rightarrow \operatorname{gr}(A \cap \mathcal{N}) \rightarrow \operatorname{gr}(A) \rightarrow g r_{F}(A+\mathcal{N}) \rightarrow 0
$$

associated to the filtration $\tau_{\mathcal{N}}=\tau^{\cdot}(A) \cap \mathcal{N}$ of $A \cap \mathcal{N}$, the filtration $\tau^{\cdot}(A)$ of $A$ and the filtration $F^{\cdot}=\tau^{\cdot}(A)+\mathcal{N}$ of $A+\mathcal{N}$. Note that, by Proposition 3.4, $\tau_{\mathcal{N}}=\tau^{\cdot}(A) \cap \mathcal{N}$. The sequence is exact in each degree, so that

$$
\left|\tau_{\mathcal{N}}^{i} / \tau_{\mathcal{N}}^{i+1}\right|+\left|F^{i} / F^{i+1}\right|=\left|\tau^{i} / \tau^{i+1}\right|
$$

Multiply this by $p^{-i n}$, and add for all $i \geqslant 0$. Then by the definition of $t$, (Definition 3.3.1) it is clear that

$$
t(A)=t_{\mathcal{N}}(A \cap \mathcal{N})+t_{F}(A+\mathcal{N}) .
$$

This together with the inequality $t_{F}(A+\mathcal{N}) \geqslant t_{\mathcal{M} / \mathcal{N}}(A+\mathcal{N})$ (Lemma 3.3.1), proves the following corollary.

\section{Corollary 2.5.1.}

$$
t(A) \geqslant t_{\mathcal{N}}(A \cap \mathcal{N})+t_{\mathcal{M} / \mathcal{N}}(A+\mathcal{N}) .
$$

\section{Filtration holonomic modules in the affine case}

\subsection{Definition}

Definition 3.0.1. Let $X=\mathbf{A}^{n}=\operatorname{spec} k[x] . A \mathcal{D}_{X}$-module $\mathcal{M}$ is called filtration holonomic if there is a sequence $A_{i}, i=0,1 \ldots$ of finite-dimensional vector subspaces of $\mathcal{M}$ such that each element in $\mathcal{M}$ is contained in all but a finite number of $A_{i}$, and there is an integer $K$ such that $t\left(A_{i}\right) \leqslant K$ for all $i \geqslant 0$.

Note that in particular $\cup_{i \geqslant 0} A_{i}=\mathcal{M}$.

The following proposition gives some equivalent characterizations of this concept.They are rather similar. In particular, it is technically convenient not to demand in the definition that $A_{i} \subset A_{i+1}$. However, it is shown in the proposition that it is always possible for a filtration holonomic module to find a sequence which satisfies this stricter condition.

Proposition 3.1. Let $X=\mathbf{A}^{n}=\operatorname{speck}[x]$. For a $\mathcal{D}_{X}$-module $\mathcal{M}$ the following conditions are equivalent.

i) $\mathcal{M}$ is filtration holonomic.

ii) There exist vector subspaces $A_{i}=V_{i} B_{i}, i \geqslant 0$, where $B_{i} \subset \mathcal{M}^{(i)}$, such that $A_{i} \subset A_{i+1}$ and $\cup_{i \geqslant 0} A_{i}=\mathcal{M}$. Furthermore, for this sequence, there is an integer $K$, such that $t\left(A_{i}\right) \leqslant K$.

iii)There exist vector subspaces $B_{i j} \subset \mathcal{M}^{(j)}, i \leqslant j, j=1,2 \ldots$, and an integer $K$, such that for all $i \geqslant 0, \Sigma_{j}\left|B_{i j}\right| \leqslant K$, and such that $A_{i}=\sum_{j} V_{j} B_{i j} \subset A_{i+1}=$ $\sum_{j} V_{j} A_{i+1 j}$ and $\cup_{i} A_{i}=\cup_{i, j} V_{j} B_{i j}=\mathcal{M}$.

Sequences $A_{i}, i \geqslant 0$ of the types used in the definition or the proposition will be called generating sequences, and the minimal value possible of the integer $K$ will be called the multiplicity e $(\mathcal{M})$ of the module. (Theorem 4.3 motivates the use of this last term.) 
Proof. The equivalence between ii) and iii) is immediate. Assume iii) and define $B_{i}:=\sum_{j} V_{j-i}^{\left[p^{i}\right]} B_{i j} \subset \mathcal{M}^{(i)}$. Then $t\left(V_{i} B_{i}\right)=t_{i}\left(B_{i}\right) \leqslant \Sigma_{j}\left|B_{i j}\right|$ by Lemma 3.3 .1 and Proposition 3.3. Thus the sequence $B_{i}$ satisfies all the conditions in ii). The converse implication follows from applying Proposition 3.3. in the converse direction.

To continue, clearly ii) trivially implies i). It thus remains to prove that i) implies ii). Assume then the existence of $A_{i}$ and $K$ as in the definition of a filtration holonomic module. We claim that, for a fixed $j$, the sequence $\tau^{j}\left(A_{k}\right), k \geqslant 0$, also constitutes a generating sequence. Given an element $m \in \mathcal{M}$ there is a finitedimensional vector space $\Phi \subset \mathcal{M}^{(j)}$, such that $m \in V_{j} \Phi$ (by Proposition 2.4), and since each element of a fixed basis of $V_{j} \Phi$ is contained in almost all the $A_{i}$, $V_{j} \Phi \subset A_{k}$ for all $k$ large enough, and hence also, for these $k, m \in \tau^{j}\left(A_{k}\right)$. This shows that each element in $\mathcal{M}$ is contained in almost all $\tau^{j}\left(A_{k}\right), k \geqslant 0$. Also, for a finite-dimensional vector space $A, \tau^{k}\left(\tau^{j}(A)\right)=\tau^{\max \{j, k\}}(A)$ and hence $t(A)=$ $\sum_{k \geqslant 0} p^{-k n}\left|\tau^{k}(A) / \tau^{k+1}(A)\right| \geqslant \sum_{k \geqslant j} p^{-k n}\left|\tau^{k}(A) / \tau^{k+1}(A)\right|=t\left(\tau^{i}(A)\right.$ (Definition 3.2) and in particular, for all $k \geqslant 0$, we have $t\left(\tau^{j}\left(A_{k}\right)\right) \leqslant K$. Thus the claim is proved. Note that since any element in $\mathcal{M}$ is contained in almost all the vector spaces of a generating sequence, it is clear that any finite-dimensional vector subspace of $\mathcal{M}$ is also contained in almost all elements of the generating sequence. This applies then in particular to the sequence $\tau^{j}\left(A_{k}\right), k \geqslant 0$, for any fixed $j \geqslant 0$. Now consider the double sequence $\tau^{j}\left(A_{k}\right), k, j \geqslant 0$. Each of these vector spaces has $t \leqslant K$. Choose recursively a diagonal subsequence $C_{i}=V_{i} B_{i}, i \geqslant 0$, where $B_{i} \subset \mathcal{M}^{(i)}$ in the following way. First set $C_{0}:=A_{0}$. If $C_{i}=V_{i} B_{i}$, where $B_{i} \subset \mathcal{M}^{(i)}$ has been chosen for $i \leqslant i_{0}$, then consider the sequence $\tau^{i_{0}+1}\left(A_{k}\right), k \geqslant 0$, and choose as $C_{i_{0}+1}$ any one of these spaces which contains both $C_{i_{0}}$ and $A_{i_{0}+1}$ (This is possible by the preceding argument). Each vector space in the sequence $\tau^{i_{0}+1}\left(A_{k}\right), k \geqslant 0$ is of the form $V_{i_{0}+1} B$, for some vector space $B \subset \mathcal{M}^{\left(i_{0}+1\right)}$, by Lemma 3.2.1. Hence $C_{i}=V_{i} B_{i}, i \geqslant 0$, where $B_{i} \subset \mathcal{M}^{(i)}$. From the fact $A_{i} \subset C_{i}$ for all $i \geqslant 0$, we see that the union of $C_{i}$ is $\mathcal{M}$. Also we just saw that $t\left(C_{i}\right) \leqslant K$, for all $i \geqslant 0$, and $C_{i} \subset C_{i+1}$, by construction and hence $B_{i}$, satisfies all the properties of ii).

Examples are given in section 5 .

\subsection{Fundamental properties in the case $X=\mathbf{A}^{n}$}

Theorem 3.2. Submodules, quotient and extensions of filtration holonomic modules are filtration holonomic, and every filtration holonomic module has a finite decomposition series. The number of simple quotients in a decomposition series is bounded by $e(\mathcal{M})$.

Proof. Let $\mathcal{M}$ be a filtration holonomic $\mathcal{D}_{X}$-module, with $A_{i}, i \geqslant 0$ as a generating sequence with $t\left(A_{i}\right) \leqslant K$, for all $i \geqslant 0$ as in the definition. Suppose first that $\mathcal{N}$ is a submodule of $\mathcal{M}$. Then an immediate consequence of $t_{\mathcal{N}}\left(A_{i} \cap \mathcal{N}\right) \leqslant t\left(A_{i}\right)$ (Proposition 3.4) is that $A_{i} \cap \mathcal{N}, i \geqslant 0$ is a generating sequence of $\mathcal{N}$; the other requirement, that every element in $\mathcal{N}$ is contained in all except a finite number of these subspaces is obvious since this was true in $\mathcal{M}$. Hence $\mathcal{N}$ is a filtration holonomic module. A similar argument using Proposition 3.5 gives the assertion on quotient modules. 
We next prove that $\mathcal{M}$ has a finite decomposition series. Assume that there are $K+1 \mathcal{D}_{X}$-submodules

$$
\mathcal{N}_{K+1} \subset \ldots \subset \mathcal{N}_{i+1} \subset \mathcal{N}_{i} \subset \ldots \subset \mathcal{N}_{1}=\mathcal{M} .
$$

Let $t\left(A_{j} \cap \mathcal{N}_{i}\right)$ denote the measure of $A_{j} \cap \mathcal{N}_{i}$ as a subspace of $\mathcal{N}_{i}$. Then for each fixed $j$, by Proposition 3.4, $K \geqslant t\left(A_{j} \cap \mathcal{N}_{1}\right) \geqslant t\left(A_{j} \cap \mathcal{N}_{2}\right) \geqslant \ldots$, and hence, for each $j$, there are (at least) two consecutive indices $i_{j}, i_{j}+1 \in\{1, \ldots, K+1\}$ such that $t\left(A_{j} \cap \mathcal{N}_{i_{j}}\right)=t\left(A_{j} \cap \mathcal{N}_{i_{j}+1}\right)$. Hence by the same proposition $A_{j} \cap \mathcal{N}_{i_{j}}=A_{j} \cap \mathcal{N}_{i_{j}+1}$. Now vary $j$. Since there are only a finite number of possible pairs, some pair of indices $i, i+1$ will occur for an infinite number of different $j$. So the equality $A_{j} \cap \mathcal{N}_{i_{0}}=A_{j} \cap \mathcal{N}_{i_{0}+1}$ is true for an infinite set $J$ of indices $j$. But $\cup_{j \in J} A_{j}=\mathcal{M}$ and hence

$$
\mathcal{N}_{i_{0}}=\cup_{j \in J} A_{j} \cap \mathcal{N}_{i_{0}}=\cup_{j \in J} A_{j} \cap \mathcal{N}_{i_{0}+1}=\mathcal{N}_{i_{0}+1} .
$$

Thus, any chain of $\mathcal{D}_{X}$-submodules of $\mathcal{M}$ contains at most $e(\mathcal{M})$ different modules.

Next consider an extension

$$
\mathcal{N} \hookrightarrow \mathcal{M} \rightarrow \mathcal{K}
$$

of filtration holonomic $\mathcal{D}_{X}$-modules. Let $A_{i}=V_{i} \Phi_{i}, i=0,1,2 \ldots$ where $\Phi_{i} \subset \mathcal{N}^{(i)}$, be a generating sequence of $\mathcal{N}$ with $t\left(A_{i}\right) \leqslant e(\mathcal{N})$, as in Proposition 4.1 ii). Let also the sequence $B_{i}=\sum_{j} V_{j} \Psi_{i j}, i=0,1,2 \ldots$, where $\Psi_{i j} \subset \mathcal{M}^{(j)}, i \leqslant j, j=$ $1,2 \ldots$, be a generating sequence of the type in Proposition 4.1 iii), such that for all $i \geqslant 0, t\left(B_{i}\right) \leqslant \Sigma_{j}\left|\Psi_{i j}\right| \leqslant e(\mathcal{K})$. Note that, as before, each finite-dimensional vector space in $\mathcal{N}$ is contained in all except a finite number of $A_{i}$, and similarily for the other generating sequence. There is induced a canonical short exact sequence $\mathcal{N}^{(i)} \rightarrow \mathcal{M}^{(i)} \rightarrow \mathcal{K}^{(i)}$ (Proposition 2.3) and this makes it possible to lift each $\Psi_{i j}$ to a vector subspace $\tilde{\Psi}_{i j} \subset \mathcal{M}^{(j)}$, such that $\left|\tilde{\Psi}_{i j}\right|=\left|\Psi_{i j}\right|$. Define $\tilde{B}_{i}=\sum_{j} V_{j} \tilde{\Psi}_{i j}$. By construction, $t\left(\tilde{B}_{i}\right) \leqslant t\left(B_{i}\right) \leqslant e(\mathcal{K})$. We have by assumption that $B_{i} \subset B_{i+1}$. Hence $\tilde{B}_{i} \subset \tilde{B}_{i+1}+\mathcal{N}$ and there is some $j_{i+1}$ such that $\tilde{B}_{i} \subset \tilde{B}_{i+1}+A_{j_{i+1}}$. Since $j_{i+1}$ may be taken to be any large enough integer,we might clearly inductively assume that also $A_{j_{i}} \subset A_{j_{i+1}}$ and $j_{i}<j_{i+1}$, so that finally

$$
C_{i}:=\tilde{B}_{i}+A_{j_{i}} \subset C_{i+1}:=\tilde{B}_{i+1}+A_{j_{i+1}} .
$$

Hence $\cup_{i \geqslant 0} C_{i}$ is a vector space that contains $\mathcal{N}=\cup_{i \geqslant 0} A_{j_{i}}$ and projects onto $\mathcal{K}=\cup_{i \geqslant 0} B_{i}$, and it has hence to be $\mathcal{M}$. Furthermore $t\left(C_{i_{k}}\right) \leqslant e(\mathcal{N})+e(\mathcal{K})$, by the Lemma below and hence we have constructed a generating sequence for the extension $\mathcal{M}$. Note that this implies that $e(\mathcal{M}) \leqslant e(\mathcal{N})+e(\mathcal{K})$.

Lemma 3.2.1. Let $A$ and $B$ be finite-dimensional vector subspaces of $\mathcal{M}$. Then

$$
t(A+B) \leqslant t(A)+t(B) .
$$

Proof. Consider the filtration of $A+B$ defined by $F^{i}:=\tau^{i}(A)+\tau^{i}(B)$. Clearly, $F^{i} \subset \tau^{i}(A+B)$, and hence, by Lemma 3.2,

$$
t_{F}(A+B) \geqslant t(A+B)
$$

However, there is, for arbitrary finite-dimensional vector spaces

$$
A \supset A_{1}, B \supset B_{1}
$$


contained in a common vector space, an inequality

$$
\left|(A+B) /\left(A_{1}+B_{1}\right)\right| \leqslant\left|A / A_{1}\right|+\left|B / B_{1}\right|,
$$

(Divide all vector spaces by $A_{1} \cap B_{1}$; this reduces to the case that $\left|A_{1}+B_{1}\right|=\left|A_{1}\right|+$ $\left|B_{1}\right|$, and the inequality is trivial.) Hence, $\left|F^{i} / F^{i+1}\right|=\mid\left(\tau^{i}(A)+\tau^{i}(B)\right) /\left(\tau^{i+1}(A)+\right.$ $\left.\tau^{i+1}(B)\right)|\leqslant| \tau^{i}(A) / \tau^{i+1}(A)|+| \tau^{i}(B) / \tau^{i+1}(B) \mid$, and so, by considering the definition, $t_{F}(A+B) \leqslant t(A)+t(B)$. By (1) the proof of the lemma is finished.

Theorem 3.3. If $\mathcal{N} \hookrightarrow \mathcal{M} \rightarrow \mathcal{K}=\mathcal{M} / \mathcal{N}$ is a short exact sequence of filtration holonomic $\mathcal{D}_{X}$-modules, then $e(\mathcal{M})=e(\mathcal{N})+e(\mathcal{K})$.

Proof. The inequality $e(\mathcal{M}) \leqslant e(\mathcal{N})+e(\mathcal{K})$ was proven as part of the proof of Theorem 4.2. It thus remains to check the reverse inequality $e(\mathcal{M}) \geqslant e(\mathcal{N})+e(\mathcal{K})$. However, if $A_{i}, i \geqslant 0$, is a generating sequence for $\mathcal{M}$ with $t\left(A_{i}\right) \leqslant e(\mathcal{M}), i \geqslant 0$, it was proved in the proof of the first part of Theorem 4.2, that $A_{i} \cap \mathcal{N}, i \geqslant 0$ and $A_{i}+\mathcal{N}, i \geqslant 0$, are generating sequences for $\mathcal{N}$ and $\mathcal{K}$, respectively. We now need the following simple observation. Suppose that $A_{i}, i \geqslant 0$, is a generating sequence for a filtration holonomic module $\mathcal{M}$. Then $K=\liminf _{i \rightarrow \infty} t\left(A_{i}\right)$ exists, and, by considering the subsequence $A_{i_{k}}, k \geqslant 0$, containing all $A_{i}$ such that $t\left(A_{i}\right)=K$, which clearly is another generating sequence of $\mathcal{M}$, we find that

$$
\liminf _{i \rightarrow \infty} t\left(A_{i}\right) \geqslant e(\mathcal{M}) .
$$

Returning to the proof, it is clear that Corollary 3.5.1. implies that

$$
e(\mathcal{M})=\liminf _{i \rightarrow \infty} t\left(A_{i}\right) \geqslant \liminf _{i \rightarrow \infty} t_{\mathcal{N}}\left(A_{i} \cap \mathcal{N}\right)+\liminf _{i \rightarrow \infty} t_{\mathcal{M} / \mathcal{N}}\left(A_{i}+\mathcal{N}\right) .
$$

However the observation just made, shows that

$$
\liminf _{i \rightarrow \infty} t_{\mathcal{N}}\left(A_{i} \cap \mathcal{N}\right)+\liminf _{i \rightarrow \infty} t_{\mathcal{M} / \mathcal{N}}\left(A_{i}+\mathcal{N}\right) \geqslant e(\mathcal{N})+e(\mathcal{K}),
$$

and hence the proof of the theorem is finished.

It follows from the fact that a filtration holonomic module has a finite decomposition series that such a module is finitely generated. Indeed, it is in fact, cyclic. This is clear by Staffords theorem [2, Theorem 8.18] which says that if $A$ is a simple ring, which has infinite length as a left module over itself, then an $A$-module with finite decomposition series is cyclic. That $\mathcal{D}_{X}$ is simple is proved in e.g. [6], and the statement of infinite length is an excercise.(It follows also immediately from [loc.cit. 1.3.5.].) Another result that is proven precisely as in characteristic zero is that a simple module has, considered as a module over the structure ring $k[x]$, just one associated prime. (A proof is given in $[\mathbf{2}, 3.15-17]$; if $\mathcal{M}$ is a $\mathcal{D}$-module and $q \in k[x]$ is a prime ideal, just consider the subspace consisting of elements which are annihilated by some power of $q$. It is a $\mathcal{D}$-module, and from this the proof is immediate). We have thus the following proposition.

Proposition 3.4. A filtration holonomic module is cyclic. A simple $\mathcal{D}$-module has, considered as a module over the structure ring $k[x]$, just one associated prime. 


\section{Examples}

\subsection{Localisations $k[x]_{f}$ and local cohomology}

First consider $k[x]=k\left[x_{1}, \ldots, x_{n}\right]$ itself, and take $A_{i}:=V_{i}$. Then, clearly, $\cup_{i} A_{i}=$ $k[x]$ and $t\left(A_{i}\right)=1$. Hence $k[x]$ is a filtration holonomic $\mathcal{D}_{X}$-module, and, since $e(k[x])=1$ it follows that it is simple. (This, by the way, gives an alternate proof of this simple fact.)

Next, form the localisation, $k[x]_{1+x_{1}}$, and take $A_{i}:=V_{i}\left(k+k x_{1}^{p^{i}}\right)\left(1 /\left(1+x_{1}^{p^{i}}\right)\right)$. Then $A_{i}$ contains all rational functions $p(x) /\left(1+x_{1}^{p^{i}}\right)$, where $\operatorname{deg}_{x_{j}} p(x)<p^{i}$, if $2 \leqslant j \leqslant n$, and $\operatorname{deg}_{x_{1}} p(x)<2 p^{i}$. However any rational function $p(x) /\left(1+x_{1}\right)^{r}$ is contained in $A_{i}$ for $p^{i}>\max \left\{\operatorname{deg}_{x_{1}}(p(x)), r\right\}$ large enough, since $p(x) /\left(1+x_{1}\right)^{r}=p(x)\left(1+x_{1}\right)^{p^{i}-r} /\left(1+x_{1}\right)^{p^{i}}$, if $p^{i} \geqslant r$, and

$$
\operatorname{deg}_{x_{1}}\left(p(x)\left(1+x_{1}\right)^{p^{i}-r}\right)=\operatorname{deg}_{x_{1}}(p(x))+p^{i}-r<2 p^{i},
$$

if $p^{i}>\operatorname{deg}_{x_{1}}(p(x))$. Hence $k[x]_{1+x_{1}}$ is a filtration holonomic $\mathcal{D}_{X}$-module, and the mutiplicity is less than 2 , since $t\left(A_{i}\right)=2$. It is not simple (it contains $k[x]$ ), so the multiplicity has to be exactly two.

Then generalize this example to a localization $k[x]_{f}$, by taking $A_{i}=V_{i} M_{i} / f^{p^{i}}$, where $M_{i}=\sum_{\alpha} k x^{p^{i} \alpha}$ is the vector space generated by all monomials $x^{p^{i} \alpha}$, with the multi-index $\alpha$ satisfying $\alpha_{j} \leqslant \operatorname{deg}_{x_{j}} f$. The vector space dimension of $M_{i} / f^{p^{i}}$ is precisely $t\left(A_{i}\right)=\Pi_{j} \operatorname{deg}_{x_{j}} f$, and a calculation of degrees similar to the one made above, gives that, every rational function $p / f^{r}=p f^{p^{i}-r} / f^{p^{i}}$ is contained in $A_{i}$, for $i$ large enough. Namely, $A_{i}$ clearly contains all $q / f^{p^{i}}$ for which $\operatorname{deg}_{x_{j}}(q)<$ $p^{i}\left(\operatorname{deg}_{x_{j}}(f)+1\right)$ and

$$
\operatorname{deg}_{x_{j}}\left(p f^{p^{i}-r}\right) \leqslant \operatorname{deg}_{x_{j}}(p)+\left(p^{i}-r\right) \operatorname{deg}_{x_{j}}(f)<p^{i}\left(\operatorname{deg}_{x_{j}}(f)+1\right),
$$

if $p^{i}>\max \left\{r, \operatorname{deg}_{x_{j}}(p)\right\}$. Note that the estimate of the multiplicity, gives an estimate of the number of simple modules in a decomposition series. It is also interesting to note that the generating series $A_{i}=V_{i} \Phi_{i}$ (as in Proposition 4.1 ii)) has the property that $\Phi_{i}=\Phi_{1}^{\left[p^{i}\right]}$. This is not always the case.

Note in addition that, since local cohomology modules are subquotients of localizations of the type $k[x]_{f}$, it is a consequence of Theorem 4.2 that this type of modules are further examples of filtration holonomic. This result was the motivation for the present work. Even though this result will be contained in the results in later sections, we state it here for clearness, since these later results have much messier proofs, which tend to obscure the simple idea. It was first proved in $[\mathbf{3}]$.

Proposition 4.1. A localization $k[x]_{f}$ is filtration holonomic as a $\mathcal{D}_{X}$-module, with $e\left(k[x]_{f}\right) \leqslant \Pi_{j} \operatorname{deg}_{x_{j}} f$. All local cohomology modules $H_{I}^{j}(k[x])$, where $I \in k[x]$ is an ideal are filtration holonomic modules.

\section{2. Étale algebras over a localisation $k[x]_{f}$}

Suppose that $R$ is an étale ring extension of some localisation $k[x]_{f}:=k\left[x_{1}, \ldots, x_{l}\right]_{f}$. Then by section $2.4 R$ is a $\mathcal{D}_{X}$-module, with $X=\operatorname{spec} k[x]$. We now want to show that $R$ is in fact an filtration holonomic $\mathcal{D}_{X}$-module. This is a generalization of the 
previous section, and the proof is similar, but rather more complicated. First we show that $R$ may be assumed to have a special form.

\subsubsection{Reduction to a special case}

Suppose that $\cup_{i \in I} U_{i}$ is an arbitrary finite covering of specR. Then $R$ is a submodule of $\oplus_{i \in I} R_{U_{i}}$, and to conclude that $R$ is fh (we will use this as shorthand for "filtration holonomic intermittently from now on), it suffices, by Theorem 4.2, to prove that each $R_{U_{i}}$ is fh. Now we may use the fact that locally each étale morphism is standard (e.g.[11, Theorem I.3.14] to find a cover $U_{i}, i \in I$ such that each $R_{U_{i}}$ is a standard étale algebra over some $k[x]_{f_{i}}, f_{i} \in k[x]$, and hence it is enough to prove that $R$ of the following form are fh:

(a) $\quad R:=\left(k[x]_{f}[Y] / P\right)_{h}, h \in k[x][Y]$, is an extension of $k[x]_{f}, f \in k[x]$, and

$P \in k[x]_{f}[Y]$ is monic and furthermore the derivative $P^{\prime}$ (with respect to $Y$ ) is a unit in $R$. (This is precisely the definition of standard étale extension of $k[x]$.)

Denote the map $k[x]_{f}[Y]$ toR by $\theta$. We may use that the characteristic is positive to assume, in addition(after possibly further localizing), that the following condition is also satisfied:

(b) $\theta\left(k[x]_{f}[Y]\right) \subset R$ is a free $k[x]_{f}$-module with a basis $T_{1}=1, \ldots, T_{m}=Y^{m}$, such that also for all $i>0 T_{1}^{p^{i}}, \ldots, T_{m}^{p^{i}}$ form a basis of $\theta\left(k[x]_{f}[Y]\right) \subset R$.

This follows, for example, from the following argument. That $P$ is monic in a) implies the first part of b). Clearly $\theta\left(k[x]_{f}\left[Y^{p}\right]\right) \subset \theta\left(k[x]_{f}[Y]\right) \subset R$ are both finitely generated $k[x]_{f}$-modules. They actually must have the same rank or $k(x)[Y] /(P)=$ $k(x)\left[Y^{p}\right] /\left((P) \cap k(x)\left[Y^{p}\right]\right)$, as follows from e. g. surjectivity of the relative Frobenius for an étale extension([11, VI.Lemma 13.2] ). A direct argument for this is also easy to give: Let $\overline{k(x)}$ be the algebraic closure of $k(x)$. Then $k(x)[Y] /(P)$ is also an étale $k(x)$-algebra $([\mathbf{1 1}$, Proposition 3.3c] $)$ and this is equivalent to the fact that $P=\prod_{i=1}^{i=m}\left(Y-\alpha_{i}\right)$ has no multiple roots $\alpha_{i} \in \overline{k(x)}$ ([11, Prop.3.1, ex.3.4]). Hence $\overline{k(x)}[Y] /(P)$ is isomorphic as an algebra to $\overline{k(x)}^{m}$ and the operation of taking $p$-th powers is surjective since it is surjective on $\overline{k(x)}$. Thus $\overline{k(x)}[Y] /(P)$ is generated as a $\overline{k(x)}$-algebra by $Y^{p}$ and it follows immediately that also $k(x)[Y] /(P)$ is generated as a $k(x)$-algebra by $Y^{p}$. This means that $\theta\left(k[x]_{f}\left[Y^{p}\right]\right) \subset \theta\left(k[x]_{f}[Y]\right) \subset R$ have the same rank and there is then an element $f_{1} \in k[x]$ such that the two finitely generated modules coincide, after inverting $f_{1}$. Since $k[x]$ is a domain and $R$ is flat over $k[x]$, we have that $R \subset R_{f_{1}}$ (also as $\mathcal{D}_{X}$-modules ) and again by Theorem 4.2, it suffices to prove that $R_{f_{1}}$ is fh. Hence we may assume that $\theta\left(k[x]_{f}\left[Y^{p}\right]\right)=\theta\left(k[x]_{f}[Y]\right)$ and this implies that condition b) holds for $i=1$. However it then also holds for all $i \geqslant 0$ : If $Y=Q\left(Y^{p}\right) \in R$ where $Q$ is a polynomial with coefficients in $k[x]_{f}$ then, by raising this to $p$-th powers, $Y^{p}=Q_{1}\left(Y^{p^{2}}\right)$ where $Q_{1}$ has coefficients in $k[x]_{f}$ and hence $Y=Q\left(Q_{1}\left(Y^{p^{2}}\right)\right) \in \theta\left(k[x]_{f}\left[Y^{p^{2}}\right]\right)$. This proves that $\theta\left(k[x]_{f}\left[Y^{p^{2}}\right]\right)=\theta\left(k[x]_{f}\left[Y^{p}\right]\right)$ holds, implying the case $i=2$ in $\mathrm{b}$ ), and the argument may then be repeated to show that we are reduced to assuming a) and b). 


\subsubsection{Preliminaries}

If $a$ is a positive integer, let $M_{a}$ be the vector space of all polynomials in $k[x]$ which have degree less than or equal to $a$ in each variable $x_{1}, \ldots, x_{l}$. Use $D E G(f)$ to denote the least integer $a$ such that $f \in M_{a}$. The following lemma, which will be used throughout the example, should be obvious upon inspection.

Lemma 4.2.1. If $a$ and $b$ are two positive integers then

$$
M_{a} M_{b}=M_{a+b}
$$

and also

$$
M_{p^{j}-1}\left(M_{a}\right)^{\left[p^{j}\right]}=M_{(a-1) p^{j}-1} \supset M_{p^{j} a} .
$$

Using that $M_{p^{j}-1}$ is the vector space previously called $V_{j}$, this says in particular

$$
V_{s}\left(V_{k}\right)^{\left[p^{s}\right]}=V_{s+k}
$$

4.2.3. First estimates of degrees.

Denote by $T$ the vector space generated by the basis $T_{1}, \ldots, T_{m}$. There are nonnegative integers $a$ and $r$ such that

$$
T T \subset M_{a} f^{-r} T,
$$

and hence by induction,

$$
T^{n} \subset M_{(n-1) a} f^{-(n-1) r} T
$$

and finally

$$
T^{\left[p^{j}\right]} \subset T^{p^{j}} \subset M_{\left(p^{j}-1\right) a} f^{-\left(p^{j}-1\right) r} T .
$$

I want to invert this formula, while keeping track of the degrees. Let

$$
T_{i}^{p}=\sum_{k=1}^{m} g_{i k} f^{-(p-1) r} T_{k}, i=1, \ldots, m,
$$

where then $g_{i k} \subset M_{(p-1) a}$. Let the determinant of the matrix $G=\left(g_{i k}\right)$ be $g \in$ $M_{m(p-1) a}$. It is non-zero by assumption b), in fact it must be invertible in $k[x]_{f}$. Let $G^{\left[p^{j}\right]}$ denote the matrix which has elements $g_{i k}^{p^{j}}$, and if $U, V$ are two bases of $k[x]_{f}[Y]$, denote the base change matrix by $B(U, V)$, so that $B\left(T^{[p]}, T\right)=f^{-(p-1) r} G$ (by abuse of notation letting $T$ also mean the basis $\left\{T_{i}, i=1, \ldots, m\right\}$ etc.). By Cramers formula,

$$
B\left(T, T^{(p)}\right)=g^{-1} f^{(p-1) r} H,
$$

for some matrix $H=\left(h_{i j}\right)$ with $h_{i j} \in M_{(m-1)(p-1) a}$. Now

$$
B\left(T, T^{\left[p^{j}\right]}\right)=B\left(T, T^{[p]}\right) \cdot \ldots \cdot B\left(T^{\left[p^{j-1}\right]}, T^{\left[p^{j}\right]}\right),
$$

and $B\left(T^{\left[p^{j-1}\right]}, T^{\left[p^{j}\right]}\right)=B\left(T, T^{[p]}\right)^{\left[p^{j-1}\right]}$, so that

$$
B\left(T, T^{\left[p^{j}\right]}\right)=g^{-\left(p^{j-1}+\ldots+1\right)} f^{r\left(p^{j}-1\right)} H(j),
$$


where $H(j)=H H^{[p]} \ldots H^{\left[p^{j-1}\right]}$ is a matrix with entries in $M_{(m-1)\left(p^{j}-1\right) a}$. Hence the matrix $f^{r\left(p^{j}-1\right)} H(j)$ has its elements in $M_{p^{j} a_{1}}$, where $a_{1}$ is the least integer such that $a_{1} \geqslant r \operatorname{DE} G(f)+(m-1) a$ so that $p^{j} a_{1} \geqslant\left(p^{j}-1\right) r(\mathrm{D} E G(f))+(m-1)\left(p^{j}-1\right) a$. The important thing to note here is that obviously $a_{1}$ is independent of $j$.

In terms of bases this means that there is a positive integer $a_{2}$ such that, for all $j \geqslant 1$,

$$
T \subset g^{-p^{j}} M_{p^{j} a_{2}} T^{\left[p^{j}\right]}
$$

since, if $a_{2}=\operatorname{DEG}(g)+a_{1}$,

$$
\begin{aligned}
T & \subset g^{-\left(p^{j-1}+\ldots+1\right)} M_{p^{j} a_{1}} T^{\left[p^{j}\right]} \subset g^{-p^{j}} g^{p^{j}-\left(p^{j-1}+\ldots+1\right)} M_{p^{j} a_{1}} T^{\left[p^{j}\right]} \\
& \subset g^{-p^{j}} M_{p^{j} a_{2}} T^{\left[p^{j}\right]} .
\end{aligned}
$$

4.2.4. More degree calculations

In a similar way we present $h^{-n}$ : There is a description of

$$
h=\sum_{k=1}^{m} q_{k} f^{-t} T_{k}
$$

where $q_{k} \in M_{b}$ and $b$ and $t$ are positive integers. Hence

$$
h^{n}=\sum_{k=1}^{m} q_{k}(n) f^{-(n t+(n-1) r)} T_{k},
$$

for some elements $q_{k}(n) \in M_{(n-1) a+n b}$ (Using (11) in the previous subsection, in which $r$ and $a$ are defined.) Assume now that $n \leqslant p^{j}$. Then

$$
\begin{aligned}
h^{-n}=h^{-p^{j}} h^{p^{j}-n} & =h^{-p^{j}}\left(\sum_{k=1}^{m} q_{k}\left(p^{j}-n\right) f^{-\left(\left(p^{j}-n\right) t+\left(p^{j}-n-1\right) r\right)} T_{k}\right) \\
& =h^{-p^{j}} f^{-p^{j}(t+r)}\left(\sum_{k=1}^{m} q_{k}\left(p^{j}-n\right) f^{n t+(n+1) r} T_{k}\right),
\end{aligned}
$$

and hence

$$
h^{-n} \in\left(f^{t+r} h\right)^{-p^{j}} M_{p^{j} a_{3}} T
$$

if $a_{3}:=a+b+t(\mathrm{D} E G(f))+2 r(\mathrm{D} E G(f))$ is chosen to ensure the following inequality

$$
\begin{aligned}
& \mathrm{D} E G\left(q_{k}\left(p^{j}-n\right) f^{n t+(n+1) r}\right. \\
& \quad \leqslant\left(\mathrm{D} E G\left(q_{k}\left(p^{j}-n\right)\right)\right)+\left(p^{j} t+\left(p^{j}+1\right) r\right)(\mathrm{D} E G(f)) \\
& \quad \leqslant\left(p^{j}-1\right) a+p^{j} b+\left(p^{j} t+\left(p^{j}+1\right) r\right)(\mathrm{D} E G(f)) \\
& \quad \leqslant p^{j}(a+b+t(\mathrm{D} E G(f))+2 r(\mathrm{D} E G(f)))=p^{j} a_{3} .
\end{aligned}
$$

In a similar way there is some positive integer $a_{6}$, for example $a_{6}:=\mathrm{D} E G(f)$ such that if $j \geqslant 1$ and $p^{j} \geqslant k$

$$
f^{-k}=f^{-p^{j}} f^{p^{j}-k} \in f^{-p^{j}} M_{p^{j} a_{6}} .
$$


Finally I need to present $T T$ in this way: By the assumption made in the first calculations ((11) in the preceding subsection),

$$
T T \subset f^{-r} M_{a} T \subset f^{-p^{j}} f^{p^{j}-r} M_{a} T \subset f^{-p^{j}} M_{p^{j} a_{4}} T,
$$

where $p^{j}$ is supposed to be so large that it is bigger than the fix integers $r$ and $a$ and $a_{4}=(\mathrm{DE} G(f))+1$ is such that $p^{j} a_{4} \geqslant p^{j}(\mathrm{D} E G(f))+a \geqslant\left(\mathrm{D} E G\left(f^{p^{j}-r}\right)\right)+a$.

4.2.5. Combining the calculations to prove the result

Now consider the general element of $R$ as an element of $M_{c} h^{-n} f^{-k} T(c, k$ and $n$ are positive integers).

Using (12)-(17), the following is obtained:

$$
\begin{aligned}
& M_{c} h^{-n} f^{-k} T \subset M_{c}\left(f^{-p^{j}} M_{p^{j} a_{6}}\right)\left(\left(f^{t+r} h\right)^{-p^{j}} M_{p^{j} a_{3}} T\right) T \\
& \subset\left(h f^{t+r+1}\right)^{-p^{j}} M_{p^{j}\left(a_{3}+a_{6}+1\right)}\left(f^{-p^{j}} M_{p^{j} a_{4}} T\right) \\
& \subset\left(h f^{t+r+2}\right)^{-p^{j}} M_{p\left(a_{3}+a_{6}+a_{4}+1\right)} g^{-p^{j}} M_{p^{j} a_{2}} T^{\left[p^{j}\right]} \\
& =\left(g h f^{-b_{1}}\right)^{-p^{j}} M_{p^{j} a_{5}} T^{\left[p^{j}\right]},
\end{aligned}
$$

under the hypothesis that $p^{j} \geqslant \max \{n, k, a, r, c\}$, and where $a_{5}=a_{2}+a_{3}+a_{4}+a_{6}+1$ (the +1 comes from $M_{p^{j}} \supset M_{c}$ ) and $b_{1}=t+r+2$. Both $a_{5}$ and $b_{1}$ are independent of $j$. Consider the sequence of vectorspaces (with bounded dimension)

$$
\Phi_{j}:=\left(g h f^{-b_{1}}\right)^{-p^{j}}\left(M_{a_{5}}\right)^{\left(p^{j}\right)} T^{\left[p^{j}\right]} .
$$

Note that $\Phi_{j}=\Phi_{1}^{\left[p^{j}\right]}$ I finally claim that $A_{j}=V_{j} \Phi_{j}$ form a generating sequence for $R: R$ is the union of the vectorspaces $M_{c} h^{-n} f^{-k} T$ for all different positive integers $c, k$ and $n$. Now assume that $p^{j} \geqslant \max \{n, k, a, r, c\}$. Then by Lemma 5.2.1, in the preliminaries of this example, $M_{p^{j}-1}\left(M_{a_{5}}\right)^{\left[p^{j}\right]} \supset M_{p^{j} a_{5}}$ and so

$$
M_{p^{j}-1} \Phi_{j} \supset\left(g h f^{-b_{1}}\right)^{-p^{j}} M_{p^{j} a_{5}} T^{\left(p^{j}\right)} \supset M_{c} h^{-n} f^{-k} T .
$$

Thus every element of $R$ is contained in almost all of the vector spaces $A_{j}:=$ $M_{p^{j}-1} \Phi_{j}$. Hence it is clear that they form a generating sequence, and this ends the proof. Note that it is possible to estimate $t\left(A_{j}\right)=\left|\Phi_{1}\right|$. We state this result, as a proposition.

Proposition 4.2. Let $X:=\operatorname{spec} k[x]$. An étale $k[x]_{f}$-algebra $R$ is filtration holonomic as a $\mathcal{D}_{X}$-module. In fact, there is a generating sequence $A_{i}=V_{i} \Phi_{i}$, such that $\Phi_{i}=\Phi_{1}^{\left[p^{i}\right]}$, and $\left|\Phi_{i}\right| \leqslant K$, for some positive integer $K$.

Note this proposition was stated in [3], but the proof given there, while using the same ideas, is deficient, since it assumes that the extension may be restricted to a too special case. However the theorem stated there is still true, by the above proof, since a module with a generating sequence such that $\Phi_{i}=\Phi_{1}^{\left[p^{i}\right]}$ will be filtration holonomic in the sense of $[\mathbf{3}]$.

We will need the following statement on the growth of a generating sequence relative to the growth of the sequence $W^{j}$, for a vector subspace $W \in R$. 
Lemma 4.2.2. Let $R$ be an étale $k[x]$-algebra which thus, according to the preceding proposition, is a filtration holonomic $\mathcal{D}_{X}$-module. There is then a generating sequence $A_{j}=V_{j} \Phi_{j}, j \geqslant 0$, with the following property. To any finite-dimensional vector space $W$, there is a positive integer $r$ (depending on $W$ ) such that, for all integers $j \geqslant 0, W^{j} \subset A_{l(j)+r}$, where $l(j)$ is the least integer larger than or equal to $\log _{p}(j)$.

Proof. Remember that $V_{r}=M_{p^{r}-1}$. According to 5.2.1 there is $f \in k[x]$ such that $R \subset R_{f}=\left(k[x]_{f}[Y] /(P)\right)_{h}$ and $R_{f}$ satisfies conditions a) and b) of that subsection, so that according to (18) above there is a generating sequence $\tilde{\Phi}_{j}, j \geqslant 0$ of $R_{f}$ with $M_{c} f^{-k} h^{-n} T \subset M_{p^{j}-1} \tilde{\Phi}_{j}$, if $p^{j} \geqslant \max \{c, k, n\}$ and $j \geqslant j_{0}$ is large enough. There are clearly some $c, k, n$ such that $W \subset M_{c} f^{-k} h^{-n} T$. Let $p^{r}$ be the least $p$-th power such that $p^{r} \geqslant \max \{c, k, n\}$. Then $W \subset M_{c} f^{-k} h^{-n} T \subset M_{p^{r}-1} \tilde{\Phi}_{r}$ and

$$
W^{j} \subset M_{j c} f^{-j k} h^{-j n} \subset M_{p^{r} p^{l(j)-1}} \tilde{\Phi}_{r+l(j)} .
$$

But note that $\Phi_{j}:=\tilde{\Phi}_{j} \cap R^{(j)}$, is a generating sequence for the $\mathcal{D}_{X}$-submodule $R$, according to the proof of Theorem 4.2. Hence intersecting with $R=V_{l(j)+r} R^{l(j)+r}$ gives that $W^{j} \subset R \cap V_{l(j)+r} \tilde{A}_{l(j)+r}=V_{l(j)+r} A_{l(j)+r}$, according to Lemma 3.2.1 (3).

4.2.6. Invariance under étale pullback

The following lemma, to be generalized shortly, says that the étale pullback of a filtration holonomic module is filtration holonomic.

Lemma 4.2.3. Let $X:=\operatorname{spec} R \rightarrow \operatorname{spec} S \rightarrow \mathbf{A}^{n}=\operatorname{spec} k[x]$ be étale maps of affine varieties, and assume that $\mathcal{M}$ is filtration holonomic as a $\mathcal{D}_{S}$-module. Then $R \otimes_{S} \mathcal{M}$ is filtration holonomic as a $\mathcal{D}_{R}$-module.

Proof. Since $R \otimes_{k[x]} \mathcal{M} \rightarrow R \otimes_{S} \mathcal{M}$, we may, in view of Theorem 3.2.1, assume that $S=k[x]$. Let $\Phi_{i j} \subset \mathcal{M}^{(j)}, j \geqslant i \geqslant 0$, be a generating sequence for $\mathcal{M}$ of the type in Proposition 4.1 iii), so that $\cup_{i, j} V_{j} \Phi_{i j}=\mathcal{M}$, and $\sum_{j}\left|\Phi_{i j}\right| \leqslant L$, for some fix integer $L$, and all $i \geqslant 0$. By Proposition 5.2, there is also a generating sequence $\Psi_{j}$ for $R=\cup_{j} V_{j} \Psi_{j}$, and an integer $K$ such that $\left|\Psi_{j}\right| \leqslant K, j \geqslant 0$. Then $\Theta_{i j}:=V_{1}^{\left[p^{j}\right]} \Psi_{j} \otimes \Phi_{i j} \subset R^{(j)} \otimes M^{(j)}=\left(R \otimes_{k[x]} \mathcal{M}\right)^{(j)}$, satisfies $\sum_{j}\left|\Theta_{i j}\right| \leqslant K L\left|V_{1}\right|$ and furthermore

$$
\cup_{j} V_{j} \Theta_{i j}=\cup_{j}\left(V_{j} V_{1}^{\left[p^{j}\right]} \Psi_{j}\right) \otimes \Phi_{i j} \supset \sum_{j} V_{j} \Psi_{j} \otimes V_{j} \Phi_{i j} .
$$

(We have used that $V_{j} V_{1}^{\left[p^{j}\right]} \supset V_{j} V_{j}$, see Lemma 5.2.1.) If now $m=\sum_{l \in I} r_{l} \otimes m_{l} \in$ $R \otimes_{k[x]} \mathcal{M}$, and $\left\{r_{l}, l \in I\right\} \subset V_{j} \Psi_{j}$ for $j \geqslant j_{0}$ and $\left\{m_{l}, l \in \Lambda\right\} \subset \sum_{j} V_{j} \Phi_{i j}$ for $i \geqslant i_{0}$, then clearly $m \in V_{j} \Psi_{j} \otimes V_{j} \Phi_{i j}$ if $i \geqslant \max \left(i_{0}, j_{0}\right)$, since the fact that $A_{i j}=0$, if $j<i$ implies that all the $V_{j} B_{j}$ occurring in the sum will contain $\left\{r_{l}, l \in I\right\}$. Hence every element in $R \otimes_{k[x]} \mathcal{M}$ is contained in almost all the vector spaces $\sum_{j} V_{j} C_{i j}$, and these vector spaces thus satisfy the conditions of Proposition 4.1 iii). 


\section{Filtration holonomicity for smooth varieties}

If $X$ is a integral and smooth scheme of finite type over $k$ there is a covering of $X$ by open affine sets $U_{i}, i=1, \ldots, r$, with étale maps $u_{i}: U_{i} \rightarrow \mathbf{A}^{n}$. Such a map is called a system of local coordinates, and the whole system is called an atlas. It is natural to globalize the definition of filtration holonomic modules in the following way.

Definition 5.0.1. Let $X$ be an integral and smooth scheme of finite type over $k$. If there is a covering of $X$ by open affine sets $U_{i}, i=1, \ldots, r$, and étale maps $u_{i}: U_{i} \rightarrow \mathbf{A}^{n}$, such that $\left.u_{i *} \mathcal{N}\right|_{U_{i}}$ is a filtration holonomic $\mathcal{D}_{\mathbf{A}}^{n}$-module, then the $\mathcal{D}_{X}$-module $\mathcal{N} \mid$ is called filtration holonomic, in the given atlas. If this is true in one atlas, it is true in all.

We will now prove the stated independence of the atlas. The following lemma contains the main ingredient.

Lemma 5.0.4. Assume that $u, v: \operatorname{spec} R \rightarrow \mathbf{A}^{n}=\operatorname{spec} k\left[x_{1}, \ldots, x_{n}\right]$ are étale maps

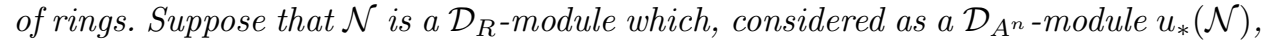
using the map $u$, is filtration holonomic. Then $v_{*} \mathcal{N}$ is also filtration holonomic.

Proof. The ring homomorphisms, corresponding to the maps $u, v$ in the lemma, will also be denoted by $u, v: k\left[x_{1}, \ldots, x_{n}\right] \rightarrow R$. The homomorphism $v$ will be identified with an inclusion $k[x] \subset R$. Since this inclusion is étale, $R$ is filtration holonomic as a $\mathcal{D}_{A^{n}}$-module by Proposition 5.2. Let $A_{s}=V_{s} \Phi_{s}, s \geqslant 0$ be a generating sequence for $R$ constructed so as to have the properties in lemma 5.2.3. We can take $W:=u\left(M_{1}\right)$ in this lemma and then obtain that there is a fixed $r$ such that for all $s \geqslant 0$ we have

$$
u\left(V_{s}\right)=u\left(M_{p^{s}-1}\right) \subset u\left(M_{1}^{p^{s}-1}\right)=u\left(M_{1}\right)^{p^{s}-1} \subset A_{s+r}=V_{s+r} \Phi_{s+r} .
$$

(Note that $l\left(p^{s}-1\right)=s$.) Let furthermore $L=e(R) \geqslant\left|\Phi_{s}\right|, s \geqslant 0$. Since $u_{*} \mathcal{N}$ is filtration holonomic there exist, according to Prop 4.1, vector subspaces $B_{i j} \subset$ $\mathcal{N}^{(j)}, 0 \leqslant i \leqslant j$, and an integer $K$, such that for all $i \geqslant 0, \sum_{j \geqslant i}\left|B_{i j}\right| \leqslant K$, and $\sum_{j \geqslant i} u\left(V_{j}\right) B_{i j} \subset \sum_{j \geqslant i} u\left(V_{j}\right) B_{i+1 j}$ and such that $\cup_{i, j \geqslant 0} u\left(V_{j}\right) B_{i j}=u_{*} \mathcal{N}$. Define now

$$
C_{i j}:=V_{r}^{\left[p^{j}\right]} \Phi_{j+r} B_{i j}
$$

Since, for all $i \geqslant 0, \mathcal{N}^{(j)}$ is an $R^{(j)}$-module, and $V_{r}^{\left[p^{j}\right]} \Phi_{j+r} \subset R^{(j)}$, it is clear that $C_{i j} \subset \mathcal{N}^{(j)}$. Let

$$
C_{i}:=\sum_{j \geqslant i} V_{j-i}^{\left[p^{i}\right]} C_{i j}
$$

Clearly, by definition, $t\left(V_{j} C_{i j}\right)=\left|\Phi_{j+r}\right|\left|B_{i j}\right| \leqslant L\left|B_{i j}\right|$ and hence

$$
t\left(V_{i} C_{i}\right) \leqslant \sum_{j \geqslant i} t\left(V_{j} C_{i j}\right) \leqslant K L
$$

is uniformly bounded for all $i$. Now

$$
V_{j} C_{i j}=V_{j} V_{r}^{\left[p^{j}\right]} \Phi_{j+r} B_{i j} \supset V_{j+r} \Phi_{j+r} B_{i j} \supset u\left(V_{j}\right) B_{i j}
$$


and this shows that $V_{i} C_{i}=\cup_{j} \geqslant i V_{j} C_{i j} \supset \cup_{j \geqslant i} u\left(V_{j}\right) B_{i j}$ and so each element in $u^{*} \mathcal{N}$ is contained in almost all of the vector spaces $V_{i} C_{i}$. (We have above repeatedly used the formula $V_{s}\left(V_{b}\right)^{\left[p^{s}\right]}=V_{b+s}$, of Lemma 3.2.2.) Hence $V_{i} C_{i}$ is a generating sequence and so the lemma is proved.

With the lemma it is easy to prove the independence of the atlas used in the definition of filtration holonomic . Assume that $\mathcal{N}$ is filtration holonomic in the atlas $u_{i}: U_{i} \rightarrow \mathbf{A}^{n}, i \in I$, and that $v_{j}: V_{j} \rightarrow \mathbf{A}^{n}, j \in J$ is another étale atlas, not necessarily affine nor covering. There is a finite covering of each $V_{j} \cap U_{i}$ by open affine subsets, $\cup_{k} W_{i j}^{k}=V_{j} \cap U_{i}$, and with induced étale affine maps $u_{i}, v_{j}$ : $W_{i j}^{k} \rightarrow \mathbf{A}^{n}, i \in I, j \in J$. By Lemma 5.2 .3 we know that $u_{i *}\left(\mathcal{N} \mid W_{i j}^{k}\right)$ is filtration holonomic as a $\mathcal{D}_{\mathbf{A}}^{n}$-module. Observe, by the way, that this shows in particular, that taking the second atlas to consist of just the inclusion of an arbitrary open subset $v: V \subset X$, we see that if $\mathcal{N}$ is a filtration holonomic $\mathcal{D}_{X}$-module then $\mathcal{N} \mid V$ is filtration holonomic . Returning to the general case, and assuming that the second atlas is affine and covering, the lemma above shows that then $v_{j *}\left(\mathcal{N} \mid W_{i j}^{k}\right)$ is filtration holonomic as a $\mathcal{D}_{\mathbf{A}^{n} \text {-module, and since }}$

$$
v_{j *}\left(\mathcal{N} \mid V_{j}\right) \subset \oplus_{k, i} v_{j *}\left(\mathcal{N} \mid W_{i j}^{k}\right),
$$

the submodule part of Theorem 4.2 gives that $v_{j *}\left(\mathcal{N} \mid V_{j}\right)$ is a filtration holonomic

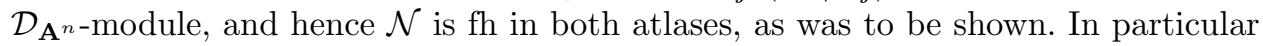
the new definition coincides with the old one for $X=\mathbf{A}^{n}$ !

In a similar way the following proposition is proved. It contains of course the earlier results in 5.1 and 5.2.6.

Proposition 5.1. Let $u: X \rightarrow Y$ be an étale map .

i) If $\mathcal{N}$ is a filtration holonomic $\mathcal{D}_{X}$-module then $u_{*}(\mathcal{N})$ is a filtration holonomic $\mathcal{D}_{Y}$-module.

ii) If $\mathcal{M}$ is a filtration holonomic $\mathcal{D}_{Y}$-module then $u^{*}(\mathcal{M})$ is a filtration holonomic $\mathcal{D}_{X}$-module.

Proof. For i), note first that, by the observation in the preceding paragraph, if $\mathcal{N}$ is a filtration holonomic $\mathcal{D}_{X}$-module and $V \subset X$ is an arbitrary open subset then $\mathcal{N} \mid V$ is filtration holonomic. Hence i) is local in $Y$, so we may assume that $Y=\mathbf{A}^{n}$. Let $V_{j} \subset X, j \in J$ be a covering of $X$ by affine open subsets. Clearly, by the lemma above, $u_{*}\left(\mathcal{N} \mid V_{j}\right)$ is filtration holonomic, and hence $u_{*}(\mathcal{N}) \subset \oplus_{j} u_{*}\left(\mathcal{N} \mid V_{j}\right)$ is holonomic.

For ii), it suffices to note that the statement is local in both $X$ and $Y$, and hence is just a version of Lemma 5.2.6.

Finally, note that, once given the definition of filtration holonomic for arbitrary $X$, Theorem 4.2., on finite decomposition series etc. of filtration holonomic modules, still remains true, substituting $X$ for $\mathbf{A}^{n}$, by a trivial argument. This is Theorem 1 of the introduction. 


\section{Functoriality}

Haastert [5] has defined inverse and direct images of $\mathcal{D}_{X}$-modules in positive characteristics, and we will prove in this section that these functors preserve filtration holonomicity. He has also proven the precise analogue of Kashiwara's theorem on $\mathcal{D}$-modules with support on a closed, smooth subvariety, and we prove that this correspondence also preserves filtration holonomicity. Though the theory in $[\mathbf{5}]$ is stated for smooth varieties over an algebraically closed field, it is clear from the definitions (given below), that they work for an arbitrary field.

\subsection{Maps}

Suppose then that $f: X \rightarrow Y$ is a map between two smooth varieties $X$ and $Y$. Then $f$ may be factored into a closed immersion $\Gamma_{f}: X \rightarrow X \times Y$ ([EGA I.5.2.4]), using that the schemes are separated, followed by the projection $X \times Y \rightarrow Y$. For a closed immersion of a smooth subvariety in a smooth variety, there is the following very nice description ([SGA 1:II.4.9-10]).

Lemma 6.1.1. Let $X$ be a smooth $k$-scheme, with a closed subscheme $Y$, and let $x$ be a point in $X$. If $Y$ is smooth, then there is an open neighbourhood $x \in X_{1} \subset X$ and an étale morphism

$$
g: X_{1} \rightarrow X_{2}=\operatorname{speck}\left[t_{1}, \ldots, t_{n}\right]
$$

such that the closed subset $Y_{1}=Y \cap X_{1} \subset X_{1}$ is the inverse image by $g$ of the closed subscheme $Y_{2}=\operatorname{speck}\left[t_{r+1}, \ldots, t_{n}\right]$, for some suitable $r$. In particular the restricted map $g: Y_{1} \rightarrow Y_{2}$, is étale.

\subsection{Inverse images}

If $f: X \rightarrow Y$ is a map between two smooth varieties $X$ and $Y$, the inverse image of a $\mathcal{D}_{Y}$-module $\mathcal{M}$ is, as an $\mathcal{O}_{X}$-module simply $f^{*} \mathcal{M}$, i.e.the ordinary pullback as a quasi-coherent $\mathcal{O}_{X}$-module $([\mathbf{5}, 2.1])$. To explicit the differential structure, assume that the inverse system belonging to $\mathcal{M}$ is $\mathcal{M}^{(r)} \supset \mathcal{M}^{(r+1)}$, where $r \geqslant 0$. The corresponding inverse system for $f^{*} \mathcal{M}$ is given by $f^{(r) *} \mathcal{M}^{(r)}$, where $f^{(r)}: X^{(r)} \rightarrow Y^{(r)}$ is the induced map. The injection $f^{(r+1) *} \mathcal{M}^{(r+1)} \hookleftarrow f^{(r) *} \mathcal{M}^{(r)}$, is the composition

$$
\mathcal{O}_{X^{(r+1)}} \otimes_{\mathcal{O}_{Y}(r+1)} \mathcal{M}^{(r+1)} \rightarrow \mathcal{O}_{X^{(r)}} \otimes_{\mathcal{O}_{Y}(r+1)} \mathcal{M}^{(r)} \rightarrow \mathcal{O}_{X^{(r)}} \otimes_{\mathcal{O}_{Y}(r)} \mathcal{M}^{(r)}
$$

where the first map is the tensor product over of the canonical injections $\mathcal{O}_{X^{(r+1)}} \subset$ $\mathcal{O}_{X^{(r)}}$, and $\mathcal{M}^{(r+1)} \subset \mathcal{M}^{(r)}$, and the second map is the canonical surjection. Recall also that if $s: X \rightarrow Y$ is an étale map then the direct image $s_{*}(\mathcal{M})$ of the $\mathcal{D}_{X^{-}}$ module $\mathcal{M}$ considered as a quasi-coherent module is a $\mathcal{D}_{Y}$-module.

Proposition 6.1. Let $f: X \rightarrow Y$ be a morphism, and $\mathcal{M}$ a filtration holonomic $\mathcal{D}_{Y}$-module. Then the inverse image $f^{*} \mathcal{M}$ is filtration holonomic.

Proof. The statement is local in both $X$ and $Y$, and it is thus enough to prove the proposition when $f$ is a morphism of affine schemes. According to 7.1, it may be assumed to be either a closed immersion or a projection. Assume first that the map $f$ is a closed immersion $X \rightarrow Y$ and use the lemma in 6.1, to obtain a diagram 


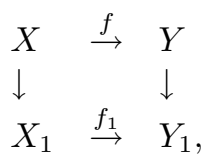

where the vertical arrows $\beta: Y \rightarrow Y_{1}=\operatorname{speck}\left[t_{1}, \ldots, t_{n}\right]$ and $\alpha: X \rightarrow X_{1}=$ speck $\left[t_{r+1}, \ldots, t_{n}\right]$ are étale maps, and $I=\left(t_{1}, \ldots, t_{r}\right)$ is the ideal which defines $X_{1} \subset Y_{1}$. We have, according to the definition, to show that $\alpha_{*} f^{*}(\mathcal{M})$ is a filtration holonomic $\mathcal{D}_{X_{1}}$-module. But clearly

$$
\alpha_{*} f^{*}(\mathcal{M}) \cong f_{1}^{*} \beta_{*}(\mathcal{M})=\beta_{*}(\mathcal{M}) / I \beta_{*}(\mathcal{M}),
$$

since both the vertical arrows are étale, and it is enough to prove that this last module is filtration holonomic. But this is almost immediate: By assumption, $\beta_{*}(\mathcal{M})$ is filtration holonomic ; let $A_{i} \subset A_{i+1}, i \geqslant 0$ be a generating sequence, so that $\beta_{*}(\mathcal{M})=\cup_{i \geqslant 0} A_{i}$. Then $\beta_{*}(\mathcal{M}) / I \beta_{*}(\mathcal{M})=\cup_{i \geqslant 0} \bar{A}_{i}$, where $\bar{A}_{i}:=A_{i}+I \mathcal{M}$. Also $\bar{A}_{i} \subset \bar{A}_{i+1}$. Thus, it suffices to check that, $t\left(\bar{A}_{i}\right) \leqslant t\left(A_{i}\right)$. But if $A_{i}=\sum_{j} V_{j}^{Y_{1}} \Phi_{i j}$, where $V_{j}^{Y_{1}}$ is the monomials of degree less than $p^{i}-1$ in the variables $t_{1}, \ldots, t_{n}$, then $\bar{A}_{i}=\sum_{j} V_{j}^{X_{1}} \bar{\Phi}_{i j}$, where $V_{j}^{X_{1}}=V_{i}^{X_{1}} \bmod I$, is the monomials of degree less than $p^{i}-1$ in the variables $t_{r+1}, \ldots, t_{n}$, and where $\bar{\Phi}_{i j} \subset\left(\beta_{*}(\mathcal{M}) / I \beta_{*}(\mathcal{M})\right)^{(i)}$. If $\Phi_{i j}$ have been chosen so that $\sum_{j}\left|\Phi_{i j}\right|=t\left(A_{i}\right)$, it follows from Proposition 4.1. that $t\left(\bar{A}_{i}\right) \leqslant$ $\sum_{j}\left|\bar{\Phi}_{i j}\right| \leqslant t\left(A_{i}\right)$. We have thus proved that $\beta_{*}(\mathcal{M}) / I \beta_{*}(\mathcal{M})$ is filtration holonomic, and this concludes the proof of the proposition in the case of an immersion.

The argument in the case of a projection is entirely similar; by suitable étale maps it is possible to assume that $f$ is the projection $X=\operatorname{spec} k[x] \times_{\text {speck }} \operatorname{spec} k[y] \rightarrow$ $Y=\operatorname{spec} k[y]$. Then $f^{*} \mathcal{M}=k[x] \otimes_{k} \mathcal{M}$ (with the obvious action of the differential operators). Let $V_{j}^{y}\left(V_{j}^{x}, V_{j},\right)$ be the monomials of degree less than $p^{i}-1$ in the $y(x, x$ and $y)$-variables, respectively. If $A_{i}=\sum_{j} V_{j}^{y} \Phi_{i j}$, is a generating sequence for $\mathcal{M}$, for which $A^{i} \subset A^{i+1}$ then clearly

$$
f^{*} \mathcal{M}=k[x] \otimes_{k} \mathcal{M}=k[x] \otimes_{k}\left(\cup_{i \geqslant 0} A_{i}\right)=\cup_{i \geqslant 0} V_{i}^{x} A_{i} .
$$

Since

$$
V_{i}^{x} A_{i}=\sum_{j} V_{i}^{x} V_{j}^{y} \Phi_{i j} \subset \tilde{A}_{i}:=\sum_{j} V_{j}^{x} V_{j}^{y} \Phi_{i j}=\sum_{j} V_{j} \Phi_{i j}
$$

and obviously $t\left(\tilde{A}_{i}\right) \leqslant \sum_{j}\left|\Phi_{i j}\right|=t\left(A_{i}\right)$ (by assumption), clearly $\tilde{A}_{i}, i \geqslant 0$, is a generating sequence for $f^{*} \mathcal{M}$, which thus is filtration holonomic. This finishes the proof. It may be noted that it follows clearly from the proof that the multiplicity does not increase.

\subsection{Direct images}

We will now consider direct images. Given a morphism $f: X \rightarrow Y$, there is a left $\mathcal{D}_{X}-f^{-1} \mathcal{D}_{Y}$-bimodule $\mathcal{D}_{Y \leftarrow X}$ and the direct image $\int_{f} \mathcal{M}$ of a $\mathcal{D}_{X}$-module $\mathcal{M}$ is defined as $f_{*}\left(\mathcal{D}_{Y \leftarrow X} \otimes_{\mathcal{D}_{X}} \mathcal{M}\right)$. It is a left exact functor and the derived functors are just $R^{i} f_{*}\left(\mathcal{D}_{Y \leftarrow X} \otimes_{\mathcal{D}_{X}} \mathcal{M}\right), i \geqslant 0$. The definition of $\mathcal{D}_{Y \leftarrow X}$ involves the canonical bundle $\omega$ over $X$ and $Y$, due to the fact that one has to make a detour over right 
$\mathcal{D}_{X}$-modules, and $\omega$ is the archetypical right $\mathcal{D}_{X}$-module. A right $\mathcal{D}_{X}$-module $\mathcal{N}$ may be described as a direct system of $\mathcal{O}_{X^{(r)}}$-modules $\mathcal{N}^{(r)}, r \geqslant 0$, with $\mathcal{O}_{X^{(r+1)}}$ -homomorphisms $\mathcal{N}^{(r)} \rightarrow \mathcal{N}^{(r+1)}$, such that the induced homomorphism

$$
\mathcal{N}^{(r)} \rightarrow \mathcal{H o m}_{\mathcal{O}_{X}(r)}\left(\mathcal{O}_{X}, \mathcal{N}^{(r+1)}\right)
$$

is an isomorphism. The left $\mathcal{D}_{X^{(r)}}$-module structure of $\mathcal{O}_{X}$ induces a right $\mathcal{D}_{X^{(r)}}$ module structure on $\mathcal{H o m}_{\mathcal{O}_{X}(r)}\left(\mathcal{O}_{X}, \mathcal{N}^{(r+1)}\right)$, and this transports by the isomorphism to a right $\mathcal{D}_{X^{(r)}}$-module structure on $\mathcal{N}^{(r)}$. Given a right $\mathcal{D}_{X}$-module $\mathcal{N}$ we have $\mathcal{N}^{(r)}=\mathcal{N} \otimes_{\mathcal{D}_{X}(r)} \mathcal{O}_{X}$, and conversely $\mathcal{N}$ may be reconstructed as

$$
\mathcal{N}=\mathcal{H o m}_{\mathcal{O}_{X}^{(r)}}\left(\mathcal{O}_{X}, \mathcal{N}^{(r)}\right)
$$

All this follows by Morita equivalence.

That $\omega_{X}$ is a right $\mathcal{D}_{X}$-module, may then be seen through the theory of local duality for finite and smooth morphisms $([\mathbf{7}$, II.6.4, 8.4]), which gives isomorphisms

$$
\operatorname{Hom}_{\mathcal{O}_{X}(r)}\left(\mathcal{O}_{X}, \omega_{X^{(r)}}\right) \cong\left(F^{(r)}\right)^{!} \omega_{X^{(r)}} \cong \omega_{X} \text {. }
$$

This is a variant of the Cartier operator, and it may locally, for a global system of local coordinates, $X=\operatorname{spec} k\left[x_{1}, \ldots, x_{n}\right]$, be described explicitly in the following way. The volume form $\eta=\wedge d x_{i}$, gives an isomorphism $k[x] \cong \omega_{X}, a \mapsto a \eta$, and $k\left[x^{p^{r}}\right] \cong \omega_{X^{(r)}}, a \mapsto a(\eta)^{p^{r}}$. Define the $k\left[x^{p^{r}}\right]$-linear map $C: \omega_{X^{(r)}} \rightarrow \omega_{X^{(s)}} s>r$, by

$$
\left.C\left(x^{\alpha}(\eta)^{p^{r}}\right)=\delta_{p^{s-r}-1, \alpha}(\eta)^{p^{s}}\right),
$$

where Kronecker's symbol $\delta_{p^{s-r}-1, \alpha}=1$, if $\alpha=\left(p^{s-r}-1, p^{s-r}-1, \ldots, p^{s-r}-1\right)$, and vanishes otherwise. This map induces as above a right $\mathcal{D}_{X}{ }^{(r)}$-structure on $\omega_{X}$. It is actually determined by

$$
(a \eta) g=(-1)^{j}(g a) \eta \text { if } g=D_{x_{i}}^{(j)},
$$

and by $(a \eta) g=(g a) \eta$ if $g \in \mathcal{O}_{X}$, as in characteristic zero. (For all this see [5, 4-5].) Now return to the general situation of a morphism $f: X \rightarrow Y$ and define

$$
\mathcal{D}_{Y \leftarrow X}=\lim _{r \rightarrow \infty} \operatorname{Hom}_{f^{-1}\left(\mathcal{O}_{Y}(r)\right)}\left(f^{-1}\left(\omega_{Y}\right), \omega_{X}\right) .
$$

This is clearly a $\mathcal{D}_{Y}{ }^{(r)}-f^{-1}\left(\mathcal{D}_{X}{ }^{(r)}\right)$ - bimodule. Since, by Morita equivalence, $\mathcal{M} \cong$ $\mathcal{O}_{X} \otimes_{\mathcal{O}_{X}(r)} \mathcal{M}^{(r)}$ and $\omega_{X^{(r)}}=\omega_{X} \otimes_{\mathcal{D}_{X}(r)} \mathcal{O}_{X}$, it is easy to check that

$$
\omega_{X^{(r)}} \otimes_{\mathcal{O}_{X^{(r)}}} \mathcal{M}^{(r)}=\omega_{X} \otimes_{\mathcal{D}_{X}(r)} \mathcal{M}
$$

and hence

$$
\begin{aligned}
\int_{f} \mathcal{M} & =f_{*}\left(\mathcal{D}_{Y \leftarrow X} \otimes_{f^{-1}\left(\mathcal{D}_{X}\right)} \mathcal{M}\right) \\
& \cong \lim _{r \rightarrow \infty} f_{*}\left(\mathcal{H o m}_{f^{-1}\left(\mathcal{O}_{Y}^{(r)}\right)}\left(f^{-1}\left(\omega_{Y}\right), \omega_{X^{(r)}} \otimes_{\mathcal{O}_{X}(r)} \mathcal{M}^{(r)}\right)\right) .
\end{aligned}
$$

The maps

$$
\begin{gathered}
f_{*}\left(\mathcal{H o m}_{f^{-1}\left(\mathcal{O}_{Y}^{(r)}\right)}\left(f^{-1}\left(\omega_{Y}\right), \omega_{X^{(r)}} \otimes_{\mathcal{O}_{X}(r)} \mathcal{M}^{(r)}\right)\right) \\
\rightarrow f_{*}\left(\mathcal{H o m}_{f^{-1}\left(\mathcal{O}_{Y}^{(r+1)}\right)}\left(f^{-1}\left(\omega_{Y}\right), \omega_{X^{(r+1)}} \otimes_{\mathcal{O}_{X}(r+1)} \mathcal{M}^{(r+1)}\right)\right)
\end{gathered}
$$


between the modules in this direct limit, are induced by

$$
\begin{aligned}
& \omega_{X^{(r)}} \otimes_{\mathcal{O}_{X^{(r)}}} \mathcal{M}^{(r)} \cong \omega_{X^{(r)}} \otimes_{\mathcal{O}_{X^{(r)}}} \mathcal{O}_{X^{(r)}} \otimes_{\mathcal{O}_{X^{(r+1)}}} \mathcal{M}^{(r+1)} \\
& \cong \omega_{X^{(r)}} \otimes_{\mathcal{O}_{X^{(r+1)}}} \mathcal{M}^{(r+1)} \stackrel{C \otimes I d}{\longrightarrow} \omega_{X^{(r+1)}} \otimes_{\mathcal{O}_{X^{(r+1)}}} \mathcal{M}^{(r+1)}
\end{aligned}
$$

Here $C$ is the Cartier operator, defined above.

$\mathcal{D}_{Y \leftarrow X}$ is a flat right $\mathcal{D}_{X}$-module, so $\int_{f}$ is left exact and has hence higher direct images

$$
\int_{f}^{i} \mathcal{M}=R^{i} f_{*}\left(\mathcal{D}_{Y \leftarrow X} \otimes_{f^{-1}\left(\mathcal{D}_{X}\right)} \mathcal{M}\right) .
$$

It should also be noted that in the case of an étale affine morphism, it follows from $f^{*}\left(\omega_{Y}\right)=\omega_{X}$, that the direct image

$$
\int_{f} \mathcal{M}=f_{*}\left(\mathcal{D}_{Y \leftarrow X} \otimes_{\mathcal{D}_{X}} \mathcal{M}\right)=f_{*} \mathcal{M}
$$

is just the direct image of $\mathcal{M}$ as an $\mathcal{O}_{X}$-module.

We can now prove the following

Proposition 6.2. Let $f: X \rightarrow Y$ be a morphism, and $\mathcal{M}$ a fh $\mathcal{D}_{X}$-module. Then the direct images $\int_{f}^{i} \mathcal{M}, i \geqslant 0$, are filtration holonomic.

Proof. The proposition is local in $Y$, and it may thus be assumed that $Y$ is affine. Since $X$ may be covered by a finite number $U_{1}, \ldots, U_{s}$ of affine open subsets we may use Čech cohomology to calculate $R^{i} f_{*}$. In particular $R^{i} f_{*}\left(\mathcal{D}_{Y \leftarrow X} \otimes_{\mathcal{D}_{X}} \mathcal{M}\right)$ is a subquotient of $\prod_{i=1}^{i=s} f_{*}\left(\left(\mathcal{D}_{Y \leftarrow X} \mid U_{i}\right) \otimes_{\mathcal{D}_{U_{i}}} \mathcal{M} \mid U_{i}\right)$, and hence by Theorem 4.2. it is enough to consider the case when $X=U_{i}$ also is affine. Since $f$ may be factored into a closed immersion followed by a projection, it is enough to prove the proposition for these two types of morphisms. Assume first that $f$ is as is described by the diagram in the proof of proposition 7.1. (This is clearly possible by shrinking each $U_{i}$, in the Čech complex above, if necessary.) Then by definition $\int_{f} \mathcal{M}$ is filtration holonomic iff $\int_{\beta} \int_{f} \mathcal{M}=\int_{f} \int_{\alpha} \mathcal{M}$ is filtration holonomic. Since $\int_{\alpha} \mathcal{M}=\alpha_{*} \mathcal{M}$ is filtration holonomic if $\mathcal{M}$ is, it clearly is enough to consider a special type of smooth subvariety, of the form

$$
f: X \cong \operatorname{speck}\left[x_{1}, \ldots, x_{n}\right] \rightarrow Y_{1} \cong \operatorname{speck}\left[x_{1}, \ldots, x_{n+m}\right]=X \times Z,
$$

where $Z_{2}=\operatorname{spec} k\left[x_{n+1}, \ldots, x_{n+m}\right]$ i.e. $f$ is the inclusion of the linear smooth subvariety, defined by the equations $x_{i}=0, i=n+1, \ldots, m+n$.

Suppose first, slightly more generally, that $f=f_{1} \times f_{2}: X \times X_{2} \rightarrow X \times Y_{2}$. Then it is easy to deduce from the description above that $\int_{f}=\int_{f_{1}} \times \int_{f_{2}}$. In our case $\int_{f} \mathcal{M}=I d_{*} \mathcal{M} \otimes_{k} \int_{f_{2}}(k)$, where $f_{2}$ is the inclusion of a point $X_{2}=$ speck in $Y_{2}$. By definition

$$
\int_{f_{2}} k=\lim _{r \rightarrow \infty} \operatorname{Hom}_{f_{1}^{-1}\left(\mathcal{O}_{Y_{2}^{(r)}}\right)}\left(f_{1}^{-1}\left(\omega_{Y_{2}}\right), \mathcal{O}_{X_{2}}\right)
$$


Since $\omega_{Y_{2}}=k\left[y^{p^{r}}\right] V_{r} \eta$ (remember that $V_{r}^{y}$ are the monomials of degree less than $p^{r}-$ 1 in each variable), $\operatorname{Hom}_{f_{1}^{-1}\left(\mathcal{O}_{Y_{2}(r)}\right)}\left(f_{1}^{-1}\left(\omega_{Y_{2}}\right), \mathcal{O}_{X_{2}}\right)=\left(V_{r}^{y} \eta\right)^{*}$. Consider the element $\phi_{r} \in\left(V_{r}^{y} \eta\right)^{*}: \phi_{r}\left(y^{\alpha}\right)=\delta_{p^{s}-1, \alpha}$ (where $\alpha$ is a multi-index, and $\delta$ as in the discussion of Cartier's operator). Then if $v \in V_{r}^{y},\left(D_{y_{i}}^{(j)} \phi_{r}\right)(v \eta)=(-1)^{j} \phi_{r}\left(D_{y_{i}}^{(j)} v \eta\right)=0$, so $\phi_{r} \in$ $\int_{f_{2}} k$ is a horizontal section for $\mathcal{D}_{Y_{2}^{(r)}}$. Clearly also $\left(V_{r}^{y} \eta\right)^{*}=V_{r}^{y} \phi_{r}$, and hence these vector spaces are a generating sequence of $\left(f_{1}\right)_{*}(k)$. If $A_{i} \cup_{j} V_{j}^{x} \Phi_{i j}$, is a generating sequence for $\mathcal{M}$, as in Proposition 4.1. iii), then $\left(f_{1}\right)_{*}(k) \times \mathcal{M}=\cup_{i j} V_{j}^{y} \phi_{r} V_{j}^{x} \Phi_{i j}$ shows that the direct image is filtration holonomic, with a generating sequence $\tilde{A}_{i}=\cup_{j} V_{j}^{y} \phi_{r} V_{j}^{x} \Phi_{i j}$. Note also that the argument gives that $e\left(\int_{f} \mathcal{M}\right) \leqslant e(\mathcal{M})$. This proves the proposition in the case of a smooth subvariety.

Suppose next that

$$
f: X \times Y \cong \operatorname{spec} k\left[x_{1}, \ldots, x_{n}, y_{1}, \ldots, y_{m}\right] \rightarrow X \cong \operatorname{speck}\left[x_{1}, \ldots, x_{n}\right]
$$

corresponds to the natural projection $x \times y \mapsto x$. Then $f$ may be decomposed $f=$ $I d_{X} \times f_{1}$, where $f_{1}$ is the projection to speck and

$$
\begin{aligned}
& \mathcal{D}_{X \leftarrow X \times Y}=\lim _{r \rightarrow \infty} \operatorname{Hom}_{\left(I d_{X} \times f_{1}\right)^{-1}\left(\mathcal{O}_{X}(r)\right.}\left(\left(I d_{X} \times f_{1}\right)^{-1}\left(\omega_{X}\right), \omega_{X} \otimes_{k} \omega_{Y}\right)= \\
= & \lim _{r \rightarrow \infty}\left(\operatorname{Hom}_{k}\left(k, \omega_{Y}\right) \otimes_{k} \operatorname{Hom}_{\mathcal{O}_{X}^{(r)}}\left(\omega_{X}, \omega_{X}\right)=\lim _{r \rightarrow \infty} \omega_{Y} \otimes_{k} \mathcal{D}_{X}^{(r)}=\omega_{Y} \otimes_{k} \mathcal{D}_{X} .\right.
\end{aligned}
$$

Then

$$
f_{*} \mathcal{M}=\lim _{r \rightarrow \infty}\left(\left(\omega_{Y} \otimes_{k} \mathcal{D}_{X}^{(r)}\right) \otimes_{\mathcal{D}_{Y}^{(r)} \otimes \mathcal{D}_{X}^{(r)}} \mathcal{M}\right)=\lim _{r \rightarrow \infty}\left(\left(\omega_{Y} \otimes_{\mathcal{D}_{Y}^{(r)}} \mathcal{M}\right) .\right.
$$

But $\omega_{Y} \otimes_{\mathcal{D}_{Y}(r)} \mathcal{M}=\mathcal{M} / I^{r} \mathcal{M}$, where $I^{r}=\oplus k D_{y_{i}}^{(j)}$, the sum taken for $1=$ $1 \ldots m, 1 \leqslant j<p^{r}$ ) since, as is easily seen $\omega_{Y}=\mathcal{D}_{Y^{(r)}} / I^{r} \mathcal{D}_{Y^{(r)}}$ (This follows from the trivial identity $\mathcal{O}_{Y}=\mathcal{D}_{Y^{(r)}} / \mathcal{D}_{Y^{(r)}} I^{r}$, in view of the description (1) of the right $\mathcal{D}_{Y^{(r)}}$-module structure of $\omega_{Y}$ given above). Now the proposition is easy to prove : Suppose that $\mathcal{M}=\cup V_{i}^{x} V_{i}^{y} \Phi_{i}$, is a generating sequence, showing that $\mathcal{M}$ is filtration holonomic.(Here we have decomposed $V_{i}=V_{i}^{x} V_{i}^{y}$, into $x$-variables and $y$-variables.)

Clearly $V_{i}^{y} / I^{r} V_{i}^{y}$ is one-dimensional over $k$, generated by the class of $y^{p-1}$ (multiindex), and hence $\overline{V_{i}^{x} V_{i}^{y} \Phi_{i}}=\overline{V_{i}^{x} y^{p-1} \Phi_{i}} \subset \mathcal{M} / I^{r} \mathcal{M}$. Since $\overline{y^{p-1} \Phi_{i}} \subset\left(\mathcal{M} / I^{+} \mathcal{M}\right)^{(i)}$, this implies that the direct image is fh, and that $e\left(\int_{f} \mathcal{M}\right) \leqslant e(\mathcal{M})$.

\subsection{Kashiwara's equivalence}

Suppose that $X \rightarrow Y$ is the immersion of a smooth subvariety. Kashiwara's theorem gives an equivalence between the category of $\mathcal{D}_{X}$-modules and the category

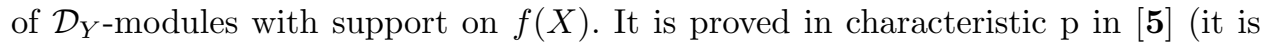
easily checked that the proof works without the hypothesis of algebraically closed ground field, since the crucial point is smoothness in the form of Lemma 7.1.1). The equivalence preserves the filtration holonomic property:

Proposition 6.3. Let $f: X \rightarrow Y$ be the immersion of a smooth subvariety. Define the functor $f^{+}$:, which takes $\mathcal{D}_{Y}$-modules to $\mathcal{D}_{X}$-modules, by

$$
f_{+}(\mathcal{M}):=f^{-1}\left(\mathcal{H o m}_{\mathcal{O}_{Y}}\left(f_{*}\left(\mathcal{O}_{X}\right), \mathcal{M}\right)\right) .
$$


Then $f^{+}:$and $\int_{f} \mathcal{M}$ gives an equivalence between the category of $\mathcal{D}_{X}$-modules and the category of $\mathcal{D}_{Y}$-modules with support on $f(X)([\boldsymbol{5}])$. The equivalence preserves the property of being fh.

Proof. By the previous section it is enough to consider the functor $f^{+}$. In terms of the description of $\mathcal{D}$-modules as inverse systems

$$
f_{+}(\mathcal{M})^{(r)}:=f^{(-1)}\left(\mathcal{H o m}_{\mathcal{O}_{Y(r)}}\left(f_{*}\left(\mathcal{O}_{X}^{(r)}\right), \mathcal{M}^{(r)}\right)\right) .
$$

The functor $f^{+}$and the statement of the proposition is local in $Y$, and so it may be assumed that there is a pullback diagram of affine schemes as in Lemma 7.1.1. Now

$$
f_{1}^{+}\left(\beta_{*} \mathcal{M}\right)=\mathcal{H o m}_{\mathcal{O}_{Y_{1}}}\left(f_{1 *} \mathcal{O}_{X_{1}}, \beta_{*} \mathcal{M}\right)=\alpha_{*} \mathcal{H o m}_{\mathcal{O}_{Y}}\left(f_{*} \mathcal{O}_{X}, \mathcal{M}\right)=\left(\alpha_{*} f^{+} \mathcal{M}\right),
$$

since $\alpha$ and $\beta$ are affine and the ideal of $X$ in $Y$ is generated by the ideal of $X_{1}$ in $Y$. Hence we are reduced to the case of $f_{1}$ and the filtration holonomic module $\beta_{*} \mathcal{M}$. Let $\mathcal{I}=\left(t_{1}, \ldots, t_{r}\right)$ be the ideal which defines $f_{1}(X)$ as a closed subset of $Y$. Then $f_{1}^{+} \beta_{*} \mathcal{M}=\left(\mathcal{I}: \beta_{*} \mathcal{M}\right)$. Denote the monomials of multi-degree less than $p^{i}-1$ in each variable $t_{1}, \ldots, t_{r}$, by $V_{i}(I)$, and denote the monomials of multi-degree less than $p^{i}-1$ in each variable $t_{r+1}, \ldots, t_{n}$, by $V_{i}(X)$. Then $V_{i}=V_{i}(X) V_{i} *(I)$. Note that $\left(\mathcal{I}: k[t] \otimes_{k\left[t^{p^{i}}\right]} \mathcal{M}^{(i)}\right)=a(i) V_{i}(X)\left(\mathcal{I}: \mathcal{M}^{(r)}\right)$, where $a(i)=\Pi_{j=1}^{r} t_{j}^{p^{i}-1}$.

Suppose that $A$ is a generating sequence for $\mathcal{N}:=\beta_{*} \mathcal{M}$, such that $A_{i} \subset A_{i+1}$, $\cup A_{i}=\mathcal{N}$ and $t_{\mathcal{N}}\left(A_{i}\right) \leqslant e$, for some fixed $e$. Then, by the lemma below, $\left(\mathcal{I}: A_{i}\right) \subset$ $\left(\mathcal{I}: A_{i+1}\right), \cup\left(\mathcal{I}: A_{i}\right)=(\mathcal{I}: \mathcal{N})$, and $t_{(\mathcal{I}: \mathcal{N}}((\mathcal{I}: A)) \leqslant e(\mathcal{N})$. So $\left(\mathcal{I}: A_{i}\right)$ is a generating sequence, and also $e(\mathcal{I}: \mathcal{N}) \leqslant e(\mathcal{N})$.

It remains to provethe following lemma.

Lemma 6.4.1. Asssume that $\mathcal{N}$ is a $\mathcal{D}_{Y}$-module, and that $A$ is a finite-dimensional vector subspace of $\mathcal{N}$. Then $t_{(\mathcal{I}: \mathcal{N})}(\mathcal{I}: A) \leqslant t_{\mathcal{N}}(A)$.

Proof. First if $B \subset A \subset \mathcal{N}$ are two finite-dimensional vector spaces contained in a $D_{Y}$-module, then $(\mathcal{I}: A) /(\mathcal{I}: B)$ maps injectively to $A / B$, since $(\mathcal{I}: A) \cap B=(\mathcal{I}$ : $B)$. Hence $|(\mathcal{I}: A) /(\mathcal{I}: B)| \leqslant|A / B|$.

Let then $F$ be any filtration of $A \subset \mathcal{N}$ such that $F^{i} \subset \tau_{\mathcal{N}}^{i}(A)$, as in Proposition 3.2. Then I claim that $G^{i}:=\left(\mathcal{I}: F^{i}\right) \subset \tau_{(\mathcal{I}: \mathcal{N})}^{i}(\mathcal{I}: A)$. This is clear since $F^{i}=$ $V_{i}(Y) \Phi^{(i)}$ implies that $\left(\mathcal{I}: F^{i}\right)=V_{i}(X) a(i)\left(I^{\left[p^{i}\right]}: \Phi^{(i)}\right)$, and $\left.a(i)\left(I^{[} p^{i}\right]: \Phi^{(i)}\right) \subset$ $((\mathcal{I}: \mathcal{N}))^{(i)}=a(i)\left(I^{\left[p^{i}\right]}: \mathcal{N}^{(i)}\right)$.

Hence it follows from the definition of $t_{F}$ in 3.2, and the first paragraph of the proof that $t_{G}(\mathcal{I}: A)=\sum p^{-n i}\left|G^{i} / G^{i+1}\right| \leqslant t_{F}(A)=\sum p^{-n i}\left|F^{i} / F^{i+1}\right|$. Since $t_{G}((\mathcal{I}$ : $A)) \geqslant t_{(\mathcal{I}: \mathcal{N})}(\mathcal{I}: A)$ (by 3.2.), it follows, by choosing $F$ so that $t_{F}(A)=t_{\mathcal{N}}(A)$, that $t_{\mathcal{M}}(A) \geqslant t_{\mathcal{I}: \mathcal{M}}(\mathcal{I}: A)$.

\section{F-modules.}

Remember that $k$ is a perfect field. Then the $p$-th power is an isomorphism of fields and hence induces an isomorphism of $\mathbf{Z}$-schemes $X^{(1)} \cong X$. This is not,in 
general, an isomorphism of $k$-schemes. Hence the following (wellknown) definition is reasonable.

Definition 7.0.1. Let $k$ be a perfect field. The $\mathcal{D}_{X}$-module $\mathcal{M}$ is called an F-module if $\mathcal{M} \cong \mathcal{M}^{(1)}$.

This thus means precisely that the modules in the inverse system belonging to the $\mathcal{D}_{X}$-module $\mathcal{M}$ are the same. From the description of the functors, in terms of inverse systems, in the preceding section, most of the following proposition is immediate.

Proposition 7.1. Fmodules are preserved by direct and inverse images, as well as by Kashiwara's functor (defined in 7.4).

Proof. Suppose that $f: X \rightarrow Y$ is a morphism. We only need to check the direct image, so let $\mathcal{M}$ be an F-module and use $\omega_{Y}=\operatorname{Hom}_{\mathcal{O}_{Y^{(1)}}}\left(\mathcal{O}_{Y}, \omega_{Y^{(1)}}\right)$ and the definition

$$
\begin{aligned}
\int_{f} \mathcal{M}: & =\lim _{r \rightarrow \infty} \operatorname{Hom}_{f^{-1}\left(\mathcal{O}_{Y}^{(r)}\right)}\left(f^{-1}\left(\operatorname{Hom}_{\mathcal{O}_{Y^{(1)}}}\left(\mathcal{O}_{Y}, \omega_{Y^{(1)}}\right)\right), \omega_{X^{(r)}} \otimes_{\mathcal{O}_{X^{(r)}}} \mathcal{M}^{(r)}\right) \\
& \cong \mathcal{O}_{X} \otimes_{\mathcal{O}_{Y^{(1)}}} \lim _{1 \leqslant r \rightarrow \infty} \operatorname{Hom}_{f^{-1}\left(\mathcal{O}_{Y}^{(r)}\right)}\left(f^{-1}\left(\omega_{Y^{(1)}}\right), \omega_{X^{(r)}} \otimes_{\mathcal{O}_{X^{(r)}}} \mathcal{M}^{(r)}\right) .
\end{aligned}
$$

(The last isomorphism is an elementary calculation). Hence clearly the direct image of an F-module is an F-module, and the proof of the proposition is finished.

\subsection{A submodule of an F-module is an F-module}

Lemma 7.1.1. Any simple subquotient $\mathcal{N}$ of a filtration holonomic F-module is a filtration holonomic F-module, possibly for a higher power of the Frobenius, i.e there is some $i \geqslant 1$ such that $\mathcal{N}=\mathcal{N}^{(i)}$.

Proof. Assume that $\theta: F^{*} \mathcal{M} \cong \mathcal{M}$ and denote the composite isomorphism $\theta \circ F^{*} \theta$ : $F^{* 2} \mathcal{M} \cong F^{*} \mathcal{M} \cong \mathcal{M}$ by $\theta_{2}$ and so on. The precise result will be that to each simple subquotient $\mathcal{L}$ there is some $\theta_{i}$ which induces an isomorphism $F^{* i} \mathcal{L} \cong \mathcal{L}$. Let $\operatorname{soc} \mathcal{M}$ be the $D$-module socle of $\mathcal{M}$. It is a semi-simple module and the same is true of $\theta\left(F^{*} \operatorname{soc} \mathcal{M}\right) \subset \mathcal{M}$, since by Cartiers lemma $F^{*} \mathcal{S}$ is simple iff $\mathcal{S}$ is. Hence $\theta\left(F^{*} \operatorname{soc} \mathcal{M}\right) \subset \operatorname{soc} \mathcal{M}$, and since these two modules have the same rank, it follows that they coincide. (If $N \cong \oplus \mathcal{L}_{i}^{n_{i}}$ where $\mathcal{L}_{i}, i=1, \ldots, r$ are distinct simple modules, the rank of $\mathcal{N}$ is $\left.n_{1}+\ldots+n_{r}\right)$. Thus soc $\mathcal{M}$ and, as a consequence, $\mathcal{M} / \operatorname{soc} \mathcal{M}$ are F-modules as well as filtration holonomic modules. By induction on the rank it then suffices to assume that $\mathcal{M}$ is semi-simple. Another application of Cartier's lemma, gives that $F^{*}$ takes distinct simple modules to distinct simple modules and hence permutes the isotypic components $\mathcal{L}_{i}^{n_{i}}$ of $\mathcal{M}$. Since the number of these are finite there is a $j$ such that $F^{* j} \mathcal{L}_{i} \cong \mathcal{L}_{i}$.

\subsection{Examples of modules that are coherent $\mathcal{O}_{X}$-modules and filtration holonomic, but not $F$-modules.}

In this section we will consider the $\mathcal{D}_{\mathbf{A}^{1} \text {-modules }} \mathcal{M}_{\alpha}$, which correspond to the differential equation $(x D) y-\alpha y=0$ with the solution $y=x^{\alpha}$. 
These modules turn out to be defined for all $\alpha \in \mathbf{Z}_{p}$ that are coherent as $k[x]$ modules, but only in very special cases F-modules. They will be the They are however always filtration holonomic.

Let $\alpha=\left(\alpha_{0}, \alpha_{1}, \ldots\right)$ be a sequence of elements in the prime field $\mathbf{Z} / p \mathbf{Z}$, and think of them as defining the element $\alpha=\alpha_{0}+\alpha_{1} p+\alpha_{2} p^{2}+\ldots$ in the p-adic completion of $\mathbf{Z}$. Using this element, define the cyclic $\mathcal{D}_{\mathbf{A}^{1} \text {-module }} \mathcal{M}_{\alpha}$ by taking $\mathcal{D}_{\mathbf{A}^{1}}=$ $k\left[x, D, D^{(p)}, D^{\left(p^{2}\right)}, \ldots\right]$ and dividing by the relations: $\alpha_{0}=x D, \alpha_{1}=x^{p} D^{(p)}, \ldots$. Call

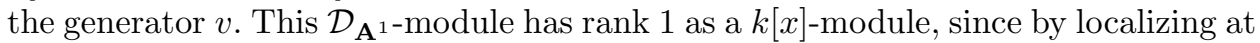
$x$, we have in it the relation $D v=\alpha_{0} x^{-1} v, D^{(p)} v=\left(\alpha_{1}\right) x^{-p} v$, etc. This also shows that $\left(\mathcal{M}_{\alpha} *\right)_{x} *=\mathcal{M}_{\alpha} *$ if $\alpha$ is not a natural number, since then there are $\alpha_{j} * \neq 0$, for arbitrarily large $j$, and hence $x^{-p^{j}} v$ will be contained in $\mathcal{M}_{\alpha} *$. Now consider the "solution set" of $\mathcal{M}_{\alpha}$ in $k[x], \operatorname{Hom}_{\mathcal{D}_{\mathrm{A}^{1}}}\left(\mathcal{M}_{\alpha}, k[x]\right)$. Since $\mathcal{M}_{\alpha}$ is a quotient of $\mathcal{D}_{\mathbf{A}^{1}}$, clearly $\operatorname{Hom}_{\mathcal{D}_{\mathbf{A}^{1}}}\left(\mathcal{M}_{\alpha}, k[x]\right) \subset \operatorname{Hom}_{\mathcal{D}_{\mathbf{A}^{1}}}\left(\mathcal{D}_{\mathbf{A}^{1}}, k[x]\right)=k[x]$, and in fact the solution set is a) at most 1-dimensional and b) represents functions $f \in k[x]$ solving the set of equations $\alpha_{1} f=x D f$ etc. Let us construct such functions. There is a change of variables involved, to $y=x-1$. First of all if $\alpha$ is a real number then there is a formal Taylor series expansion $(1+y)^{\alpha}=\sum_{i \geqslant 0}\left(\begin{array}{c}\alpha \\ i\end{array}\right) y^{i}$. For $\alpha$ a p-adic integer the right hand side still has meaning, considered as an element in the completion $\mathbf{Z}_{p}[[y]]$, if the binomial coefficients involved are defined by $\left(\begin{array}{c}\alpha \\ i\end{array}\right)=\alpha(\alpha-1) \ldots\left(\alpha_{i}+1\right) / i$ !. These binomial coefficients lie a priori in $\mathbf{Q}_{p} *$, but it is not difficult to show that they will actually be contained in $\mathbf{Z}_{p}$. By reduction to $\mathbf{Z} / p \mathbf{Z}$ we get elements $x \alpha:=(1+y)^{\alpha}$ in $\mathbf{Z} / p \mathbf{Z}[[y]]$. Now they will plausibly satisfy all properties that depends only on the binomial coefficients, and in particular they satisfy $D^{(i)} x^{\alpha}=D^{i} / i !\left(x^{\alpha}\right)=\left(\begin{array}{c}\alpha \\ i\end{array}\right) x^{\alpha-i}$ (Using that $d / d x=d / d y$ ). One has $\left(\begin{array}{l}\alpha^{i} \\ p\end{array}\right)=\alpha_{i} \in k$ by a standard identity $[6,5.1$ Identity]. Hence the element $x \alpha$ satisfies $x D x^{\alpha}=\alpha_{0} *, x^{p} D^{(p)} * x^{\alpha}=\alpha_{1}, \ldots *$ Now using $k[x] \subset k[[y]]$ we get

$$
\operatorname{Hom}_{\mathcal{D}_{\mathbf{A}^{1}}}\left(\mathcal{M}_{\alpha}, k[x]\right) \subset \operatorname{Hom}_{\mathcal{D}_{\mathbf{A}^{1}}}\left(\mathcal{M}_{\alpha}, k[[y]]\right)
$$

since we know that this last submodule is a 1-dimensional vector space (over $\mathrm{k}$ ), it must be $=k x^{\alpha}$. If there is a horisontal section of $\mathcal{M}_{\alpha}$ then the module $\operatorname{Hom}_{D}\left(\mathcal{M}_{\alpha}, k[x]\right)$ is also 1-dimensional. Hence there are horizontal sections of $\mathcal{M}_{\alpha}$ iff $x^{\alpha}$ is in $k[x]$ iff $\alpha \in \mathbf{N}$.

An isomorphism $\mathcal{M}_{\alpha} \stackrel{\eta}{\cong} \mathcal{M}_{\beta}$ occurs precisely when $\alpha=\beta+n$. Proof: localize to $\left(\mathcal{M}_{\alpha}\right)_{x} * \stackrel{\eta}{\cong}\left(\mathcal{M}_{\beta}\right)_{x} *$ considered as $k[x]_{x}$-modules. Then $\eta(v)=r(x) v, r \in k[x]_{x}$, which, by considering the solution set,implies that $r(x) x^{\beta}=x^{\alpha}$, or $r(x)=x^{n}, n \in \mathbf{Z}$ and $\alpha=\beta+n$. The converse is clear.

When is $\mathcal{M}_{\alpha}$ an $F^{r}$-module? Clearly $F^{*} \mathcal{M}_{\alpha}=\mathcal{M}_{p^{r} \alpha}$, so if this is true then $p^{r} \alpha=\alpha+n$, for some $n \in \mathbf{Z}$. This implies that $\alpha=n / p^{r}-1 \in \mathbf{Q} \cap \mathbf{Z}_{p}$. Conversely, if $\alpha \in \mathbf{Q} \cap \mathbf{Z}_{p}$, then $\alpha=n / p^{r}-1$ for some $r \geqslant 1$, and $\mathcal{M}_{\alpha}$ is an $F^{r}$-module. This means that $\mathcal{M}_{\alpha}$ is rarely an $F^{r}$-module.

$\mathcal{M}_{\alpha}$ is however always filtration holonomic, as may be seen in the following way. 
There is an isomorphism $k[[y]] \otimes_{k[x]} \mathcal{M}_{\alpha} \cong k[[y]], 1 \otimes v \mapsto x^{\alpha}$. A horizontal section in $k[[y]] \otimes_{k[x]} \mathcal{M}_{\alpha}$ is $x^{-\alpha} \otimes v$. Let $(-\alpha)_{j}=\tilde{\alpha}_{0}+\tilde{\alpha}_{1} p+\ldots+\tilde{\alpha}_{j-1} p^{j-1} \in \mathbf{Z}$, be the $j$-th truncation of the $p$-adic expansion of $-\alpha$. Then $s_{j}=x^{(-\alpha)_{j}} \otimes v=\sum_{p^{j}>i \geqslant 0}\left(\begin{array}{c}\alpha \\ i\end{array}\right) y^{i} \otimes$ $v \subset \mathcal{M}_{\alpha}$ is a $\mathcal{D}_{\mathbf{A}^{1}}^{(j)}$-horizontal section. Assume that $\alpha$ is not a natural integer (the other case is trivial). Then $p^{j}-(-\alpha)_{j}, j=1,2, \ldots$ considered as a p-adic sequence will converge to $\alpha$, and hence as a sequence of natural numbers $\lim _{j \rightarrow \infty} p^{j}-(-\alpha)_{j}=$ $\infty$. Similarily, as a sequence of natural numbers, $\lim _{j \rightarrow \infty}(-\alpha)_{j}=\infty$. Now consider $A_{j}=V_{j} s_{j} / x^{p^{j}}=V_{j} x^{-p^{j}+(-\alpha)_{j}}$. Clearly $x^{n} v \in A_{j} *$ if $(-\alpha)_{j}-1 \geqslant n \geqslant-p^{j}+(-\alpha)_{j}$, and since $\left(\mathcal{M}_{\alpha}\right)_{x}=\mathcal{M}_{\alpha} * \cong k[x]_{x}$, this implies that $\left.\mathcal{M}_{\alpha}\right)=\cup_{j} A_{j}$. Note that the multiplicity is 1 , and hence the module is simple, as a $\mathcal{D}_{X}$-module, though not finitely generated as a $k[x]$-module. However it is, of course, finitely generated as a $k[x]_{x}$-module, so these examples give some evidence towards the conjecture that all $\mathcal{O}_{X}$-coherent $\mathcal{D}_{X}$-module are filtration holonomic, especially in conjunction with the proposition in the next subsection.

\section{3. $\mathcal{O}_{X}$-coherent $\mathcal{D}_{X}$-modules $\mathcal{M}$ that are $F$-modules are filtration holo- nomic}

The following gives an alternative description of the $F$-module property for $\mathcal{O}_{X^{-}}$ coherent $\mathcal{D}_{X}$-modules; it seems to be wellknown, and is not difficult to prove.

Proposition 7.2. An $\mathcal{O}_{X}$-coherent $\mathcal{D}_{X}$-module $\mathcal{M}$ is an $F^{r}$-module iff there is an étale covering $f: Y \rightarrow X$ such that, as $\mathcal{D}_{Y}$-modules, $f^{*} \mathcal{M} \cong \oplus \mathcal{O}_{Y}$, a finite direct sum.

Proof. Suppose that the pullback to an étale covering is a finite direct sum of the structure sheaf, $f^{*} \mathcal{M} \cong \oplus \mathcal{O}_{Y}$. Then $\mathcal{M} \hookrightarrow f_{*} f^{*} \mathcal{M} \cong \oplus f_{*} \mathcal{O}_{Y}$, and $f_{*} \mathcal{O}_{Y}$ is an F-module, since, for example, trivially $F^{*} \mathcal{O}_{Y}=\mathcal{O}_{Y}$ (see 5.2.1), and direct images preserve F-modules. Hence the proposition follows from Lemma 8.1.1. Conversely, it is proved in $[\mathbf{1}, 2.17]$ that an $\mathcal{O}_{X}$-coherent $\mathcal{D}_{X}$-module $\mathcal{M}$ is locally generated by its vector space of horizontal sections $E$, over a completion $\tilde{R}_{x} \otimes_{R}$ at any point $x \in X$. Hence in particular $\mathcal{M}$ is locally free. Assume that $X=\operatorname{spec} R$ and that $\mathcal{M}=R \otimes_{k} E$ is an $F^{r}$-module, with an isomorphism, $\Theta: F^{r *} \mathcal{M} \rightarrow \mathcal{M}$ and let $e_{1}, \ldots, e_{n}$ be a basis of $E$, and $f_{i}=\sum_{j} f_{i j} e_{j}$ be a basis of the horizontal sections $F$ of $\tilde{R}_{x} \otimes_{R} \mathcal{M} . \Theta e_{i}=\sum_{j} g_{i j} e_{j}$ It is clear that $\Theta(F) \subset F$, and since $F$ and $\Theta(F)$ have the same dimension as $k$-vector spaces, it is clear that each element in $F$ is a $k$-linear combination of the elements $\Theta\left(f_{i}\right)=\sum_{j} f_{i j}^{p^{r}} \Theta\left(e_{j}\right)=\sum_{j k} f_{i j}^{p^{r}} g_{j k} e_{k}$. If $f_{j}=\sum_{i} \alpha_{j i} \Theta\left(f_{i}\right)$, then $f_{j k}=\sum_{i l} \alpha_{j i} f_{i l}^{p^{r}} g_{l k}$. Now consider the extension

$$
S=R\left[T_{i j}\right] /\left(T_{j k}-\sum_{i l} \alpha_{j i} T_{i l}^{p^{r}} g_{l k}\right) .
$$

This is, after possibly further localization of $R$, an étale extension, by the Jacobian criterion ([11][ex.3.4]). There is a map $S \rightarrow \tilde{R}_{x}$, defined by $T_{i j} \mapsto f_{i j}$, and the image $\bar{S}$ is an unramified extension of $R$, and hence, after possibly further localization of $R$, an étale extension of $R$. Hence $\bar{S} \otimes_{R} \mathcal{M} \subset \widetilde{R}_{x} \otimes_{R} \mathcal{M}$, and there are in $\bar{S} \otimes_{R} \mathcal{M}$ the horisontal sections $f_{i}=\sum_{j} T_{i j} e_{j}$. Hence $\bar{S} \otimes_{R} \mathcal{M} \cong \oplus S$, as was to be shown. Thus 
we have constructed étale morphisms $i_{x}: X_{x}=\operatorname{spec} \bar{S} \rightarrow X$ such that $i_{x}^{*} \mathcal{M}=\oplus \mathcal{O}_{X_{x}}$ By doing this at all $x \in X$, and using the quasi-compactness of $X$ to get an étale covering $Y=\amalg X_{x} \rightarrow X$.

Corollary 7.2.1. An $\mathcal{O}_{X}$-coherent $F^{r}$-module $\mathcal{M}$ is filtration holonomic.

Proof. This follows from Proposition 5.2, and Theorem 4.2.

\section{Comparison with F-finite modules}

In this section the class of filtration holonomic modules is related with the class of F-finite modules, introduced recently by G.Lyubeznik [10]. We will prove that an F-finite module is filtration holonomic .

First we will give the definition of F-finiteness. Suppose there is a finitely generated $O_{X}$-module $\mathcal{N}$ and a homomorphism $\theta: \mathcal{N} \rightarrow F_{*} \mathcal{N}$. Here we have identified $O_{X}$ with $O_{X^{(1)}}$ This homomorphism induces a direct system:

$$
\mathcal{N} \rightarrow F_{*} \mathcal{N} \stackrel{\theta}{\rightarrow} F_{*}^{2} \mathcal{N} \stackrel{F_{*} \theta}{\rightarrow} F_{*}^{3} \mathcal{N} \stackrel{F_{*}^{2} \theta}{\rightarrow} \ldots
$$

The direct limit $\mathcal{M}$ of this system is an $O_{X}$-module and F-finite modules are precisely $O_{X}$-modules that can be constructed in this way, starting with $\mathcal{N}$ and $\theta$.

Since the module automatically satisfies $\ldots F^{2 *} \mathcal{M} \cong F^{*} \mathcal{M} \cong \mathcal{M}$ it is automatically a $\mathcal{D}_{X}$-module (recall that this is equivalent to the condition that there are modules $\mathcal{M}^{(r)}$ such that $\ldots F^{2 *} \mathcal{M}^{(2)} \cong F^{*} \mathcal{M}^{(1)} \cong \mathcal{M}$, see section 1 ), in fact it is an $F$-module (see section 8 ).

For ease of notation we assume that $X=\operatorname{spec} R$ is affine. The definition of $\mathrm{F}$ finite modules works for any noetherian ring $R$, not necessarily of finite type, but we here consider only this case.

\section{Proposition 8.1. Every F-finite F-module is filtration holonomic .}

Proof. There should be a direct proof of this; the following suffices anyhow, but it uses most of the theory. Assume that the F-finite module $\mathcal{M}$ is simple - this is allowable since these modules have a finite decomposition-series by $[\mathbf{1 0}$, Theorem $3.2]$, and in view of theorem 4.2. A simple module has a unique associated prime ideal $P$ (loc.cit). There is $f \in R$ such that $(R / P)_{f}$ is non-zero regular, and hence smooth over $k$ since $\mathrm{k}$ is perfect (SGA 1 II prop.5.4). By simplicity $\mathcal{M} \subset \mathcal{M}_{f}$, and it suffices to prove that $\mathcal{M}_{f}$ is filtration holonomic (Theorem 4.2). But then we may use Kashiwaras theorem, in two different forms, to pull back the module to an $F$-module on $(R / P)_{f}$. The pullback is filtration holonomic iff if $\mathcal{M}_{f} *$ is filtration holonomic by proposition 7.2 and 7.3, and it is F-finite by [10, Proposition 3.1]. This means that it suffices to study the case $P=0$. However, there is then $f_{1} \in R$ such that $\mathcal{M} \subset \mathcal{M}_{f_{1}}$ and $\mathcal{M}_{f_{1}}$ is finitely generated as an $R_{f_{1} *}$-module (by definition), and also an $F$ - module. These modules have however been proved to be filtration holonomic in section 8 .

We will now give a partial converse. We first give a definition. If the $R$-module $\mathcal{E}$ is an $F$-module, there is an $R^{(p)}$-linear map $v: \mathcal{E} \rightarrow \mathcal{E}$ which is given by $v(m)=$ 
$\alpha(1 \otimes m)$. We will formulate a condition on generating system which means that they are invariant with respect to this Frobenius map. If the generating system $V_{i} A_{i} *$, with

$$
V_{i} A_{i} * \subset V_{i+1} A_{i+1}, t\left(A_{i}\right) \leqslant K *
$$

(where $K$ is an positive integer) and $A_{i} \subset v^{i} \mathcal{E}=\mathcal{E}^{i} \subset \mathcal{E}$, satisfies the property that

$$
v\left(A_{i}\right)=A_{i+1}, i \geqslant 1,
$$

it will be called an $F$-invariant generating system. By substituting $F^{q}$ in the above definition,instead of $F$, we get of course $F^{q}$-invariant systems. Note that then we assume that the generating system has the form $V_{i q} A_{i q}$, where $A_{i q} \subset \mathcal{E}^{i q}$. Modules with such generating systems are F-finite:

Proposition 8.2. Let the $R$-module $\mathcal{E}$ be an $F^{q}$-module $\alpha\left(F^{q}\right)^{*} \mathcal{E} \cong \mathcal{E}$, which is filtration holonomic with an $F^{q}$-invariant generating system. Then $\mathcal{E}$ is $F^{q}$-finite.

Proof. We will prove this for $q=1$. Assume that $\mathcal{E}$ is a filtration holonomic Fmodule with an isomorphism $\alpha: F^{*} \mathcal{E} \cong \mathcal{E}$, satisfying the condition on the generating system $V_{i *} A_{i}$ above. Denote the isomorphisms $\left(F^{*}\right)^{r} \mathcal{E} \cong \mathcal{E}$ by $\alpha^{(r)}$. There are, by (20) above, natural inclusions $M:=R A_{1} \subset R A_{2} \subset \ldots \mathcal{E}$, and $\mathcal{E}$ is clearly the direct limit of this direct system or more mundanely expressed $\mathcal{E}=\cup_{i \geqslant 1} R A_{i}$. Clearly $M$ is finitely generated; the number of generators is less tham $K$. We have to check that this direct system is formed in the way used for $\mathrm{F}$-finite modules. The isomorphism $\alpha: F^{*} \mathcal{E} \cong \mathcal{E}$ induces an isomorphism $t_{1}: F^{*} M=F^{*} R A_{1} \cong R v\left(A_{1}\right)=R A_{2}$ (by (21)), and hence the inclusion $i_{1}: * R A_{1} \subset R A_{2}$, induces a map $\theta_{1} *: M \rightarrow F^{*} M$. It is easy to see that more generally $\alpha^{(r)}:\left(F^{*}\right)^{r} \mathcal{E}$ congE induces $t_{r}:\left(F^{*}\right)^{r} M=$ $F^{r *} R A_{1} \cong R v^{r}\left(A_{1}\right)=R A_{r+1}$, and hence the inclusion $i_{r}: * R A_{r} \subset R A_{r+1}$, gives maps $\theta_{r} *:\left(F^{*}\right)^{r} M \rightarrow\left(F^{*}\right)^{r+1} M$. It remains only to note that $\theta_{r}=\left(F^{*}\right)^{r} \theta$, and that hence $\mathcal{E}$ is the F-finite module belonging to $M$ and $\theta$.

\subsection{Some examples of filtration holonomic modules that are not $F$ - modules.}

We will close with some examples of $D_{X}$-modules that illustrates further that the concept of filtration holonomic modules is independent of the F-module property $F^{*} \mathcal{M} \cong \mathcal{M}$. There were another in 8.2. (Though note that geometrical examples like local cohomology modules and their subquotients are $F^{r}$-modules, for some $r$ ).

The module will be constructed as a direct limit $\mathcal{M}$ of modules $A_{i}, i \geqslant 0$, each isomorphic to $k[x]$, and $k[x]$-module maps $\theta_{i}: A_{i} \rightarrow A_{i+1}$ - which are just multiplication by $\theta_{i}(1) \in k[x]$. The direct limit is thus an F-finite module if $\theta_{i}(1)^{p}=\theta_{i+1}(1)$ but not necessarily otherwise. $\mathcal{M}$ becomes a $\mathcal{D}_{k[x]}$-module, if one uses the usual $\mathcal{D}_{k[x]}$-module structure on $A_{i}$ to define the action of $D_{k[x]}^{(i)}$ in the following way. Let $x \in \mathcal{M}$ and $f \in \mathcal{D}_{k[x]}^{(i)}$; there is then some $j \geqslant i$ such that $x \in A_{j}$ and we define $f x$ using the $\mathcal{D}_{k[x]}^{(i)}$-module action on $A_{j}$. A condition making this definition welldefined is that $\theta_{i}(1) \in k\left[x^{p^{i}}\right]$. There is no need of other conditions relating the $\theta_{i}(1)$. Assume that $\mathcal{M}$ has been defined in this manner. Considered as a $\mathcal{D}_{k[x]}$-module $\mathcal{M}$ 
will be isomorphic to a submodule of the field of rational functions $k(x)$, namely the module consisting of all rational functions $p / q$ where $q$ is a product of a finite number of $\theta_{i}(1)$, each ocurring with multiplicity at most 1 . Thus it will not be possible to localise by $f \in \mathcal{M}$ to a finitely generated module if there are an infinite number of different prime divisors of the $\theta_{i}(1)$. We take, for simplicity, $\theta_{i}(1)=\left(x-a_{i}\right)^{p^{i}}$, where $a_{i}, i=0,1, \ldots$ are all different elements of the ground field $k$ (assuming that $k$ is infinite). In this case $\mathcal{M}$ is not an $F$-module, since $F * \mathcal{M}$, is isomorphic to the submodule of $k(x)$, consisting of all rational functions $p / q$ where $q$ is a product of a finite number of $\theta_{i}(1)$, each ocurring with multiplicity at most p. Any isomorphism $\eta F^{*} \mathcal{M} \cong \mathcal{M}$, will be multiplication by a rational function, and there can clearly be no such isomorphism under the assumption on the $\theta_{i}(1)$.

However the module is always filtration holonomic, if say degree $\theta_{i}(1)=p^{i}$. This is proved by the following argument. Let $s_{i}$ be the generator of $A_{i}$. Then $s_{i}=$ $\theta_{i-1}^{-1}(1) s_{i-1}$, and a generating sequence may be taken to be $V_{i} k s_{i}$. This vector space contains $V_{i-1} k s_{i-1}$, since $p^{i}-p^{i-1} \geqslant p^{i-1}$. Hence these spaces form a generating sequence, and clearly, by definition, $e(\mathcal{M})=1$, so $\mathcal{M}$ is a simple module, by the way.

\section{References}

[1] P. Berthelot and A. Ogus. Lecture notes on crystalline cohomology. Princeton UP, 1978.

[2] J.-E. Björk. Rings of Differential Operators. North-Holland, 1979.

[3] R Bogvad. Some results on D-modules in characteristic $p>0$. J. Algebra, 173:638-667, 1995.

[4] A. Borel et al. Academic Press, 1987. Lecture Notes in Mathematics, No. 20.

[5] Burkhard Haastert. On direct and inverse images of $D$-modules in prime characteristic. Manuscripta Math., 62(3):341-354, 1988.

[6] W. J. Haboush. Central differential operators on split semisimple groups over fields of positive characteristic. In Séminaire d'Algèbre Paul Dubreil et Marie-Paule Malliavin, 32ème année (Paris, 1979), volume 795 of Lecture Notes in Math., pages 35-85. Springer, Berlin, 1980.

[7] Robin Hartshorne. Residues and duality. Springer-Verlag, Berlin, 1966. Lecture Notes in Mathematics, No. 20.

[8] Craig L. Huneke and Rodney Y. Sharp. Bass numbers of local cohomology modules. Trans. Amer. Math. Soc., 339(2):765-779, 1993.

[9] Gennady Lyubeznik. Finiteness properties of local cohomology modules (an application of $D$-modules to commutative algebra). Invent. Math., 113(1):4155, 1993.

[10] Gennady Lyubeznik. F-modules: applications to local cohomology and $D$ modules in characteristic $p>0$. J. Reine Angew. Math., 491:65-130, 1997.

[11] J.S. Milne. Etale cohomology. Princeton University Press, Princeton, 1980. Lecture Notes in Mathematics, No. 169. 
[12] Karen E. Smith. The $D$-module structure of $F$-split rings. Math. Res. Lett., 2(4):377-386, 1995.

[13] Karen E. Smith and Michel Van den Bergh. Simplicity of rings of differential operators in prime characteristic. Proc. London Math. Soc. (3), 75(1):32-62, 1997.

This article may be accessed via WWW at http://www.rmi.acnet.ge/hha/ or by anonymous ftp at

ftp://ftp.rmi.acnet.ge/pub/hha/volumes/2002/n2a4/v4n2a4.(dvi,ps,pdf)

Rikard Bögvad rikard@matematik.su.se

Department of Mathematics,

University of Stockholm,

S-106 91,

Sweden 